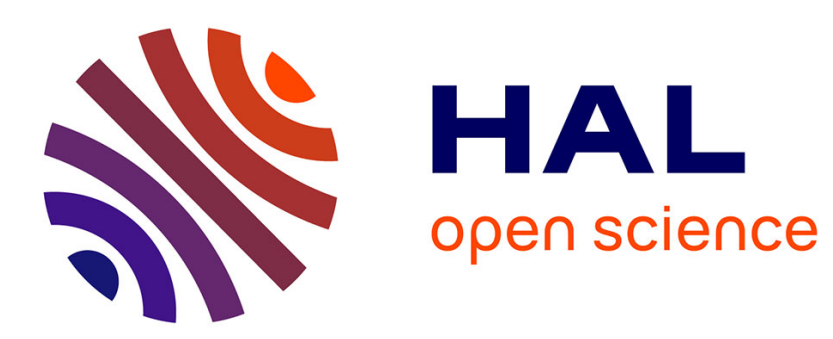

\title{
Well-posedness for a one-dimensional fluid-particle interaction model
}

Boris Andreianov, Frédéric Lagoutière, Nicolas Seguin, Takéo Takahashi

\section{To cite this version:}

Boris Andreianov, Frédéric Lagoutière, Nicolas Seguin, Takéo Takahashi. Well-posedness for a onedimensional fluid-particle interaction model. SIAM Journal on Mathematical Analysis, 2014, 46 (2). hal-00789315

\section{HAL Id: hal-00789315 https://hal.science/hal-00789315}

Submitted on 18 Feb 2013

HAL is a multi-disciplinary open access archive for the deposit and dissemination of scientific research documents, whether they are published or not. The documents may come from teaching and research institutions in France or abroad, or from public or private research centers.
L'archive ouverte pluridisciplinaire HAL, est destinée au dépôt et à la diffusion de documents scientifiques de niveau recherche, publiés ou non, émanant des établissements d'enseignement et de recherche français ou étrangers, des laboratoires publics ou privés. 


\title{
Well-posedness for a one-dimensional fluid-particle interaction model
}

\author{
Boris Andreianov ${ }^{1}$, Frédéric Lagoutière ${ }^{2}$, Nicolas Seguin ${ }^{345}$, Takéo Takahashi 678
}

\begin{abstract}
The fluid-particle interaction model introduced by the three last authors in [J. Differential Equations, 245 (2008), pp. 3503-3544] is the object of our study. This system consists of the Burgers equation with a singular source term (term that models the interaction via a drag force with a moving point particle) and of an ODE for the particle path. The notion of entropy solution for the singular Burgers equation is inspired by the theory of conservation laws with discontinuous flux developed by the first author, Kenneth Hvistendahl Karlsen and Nils Henrik Risebro in [Arch. Ration. Mech. Anal., 201 (2011), pp. 26-86]. In this paper, we prove well-posedness and justify an approximation strategy for the particle-in-Burgers system in the case of initial data of bounded variation. Existence result for $L^{\infty}$ data is also given.
\end{abstract}

Key words: Fluid-particle interaction, Burgers equation, Non-conservative coupling, Well-posedness, $B V$ estimates, Wave-front tracking, Splitting, Fixed-Point

2010 Mathematics Subject Classification: primary 35L65, secondary 35L81, 35R06, 65M12

\section{Introduction}

The aim of this paper is to prove the existence (in the $B V$ setting and in the $L^{\infty}$ setting) and uniqueness (in the $B V$ setting) for the following system that models fluid-particle interaction via drag force:

$$
\begin{aligned}
& \partial_{t} u+\partial_{x}\left(u^{2} / 2\right)=\lambda\left(h^{\prime}(t)-u\right) \delta_{0}(x-h(t)) \quad(t>0, \quad x \in \mathbb{R}), \\
& m h^{\prime \prime}(t)=\lambda\left(u(t, h(t))-h^{\prime}(t)\right) \quad(t>0)
\end{aligned}
$$

with the initial conditions

$$
u(0, x)=u_{0}(x) \quad(x \in \mathbb{R}), \quad h(0)=h_{0}, \quad h^{\prime}(0)=V_{0} .
$$

In the above one-dimensional system, $u(t, x)$ is the velocity of the fluid and we assume it satisfies the Burgers equation with a source term corresponding to the action of the particle on the fluid. The position of the particle is denoted by $h(t)$ and its motion is governed by the Newton law where we have modeled the force of the fluid on the particle by $\lambda\left(u(t, h(t))-h^{\prime}(t)\right)$, with $\lambda>0$. For more details on this model, we refer to [13] and also to [2] (see also [4] for related models).

As emphasized in [13], in the Burgers equation, the source term has to be defined: the product $u \delta_{0}$ has $a$ priori no sense since we are looking for a solution which is not continuous in variable $x$. We also need to interpret the right-hand side of the ODE $(2)$ since $u(t, \cdot)$ may be discontinuous at $x=h(t)$. Following classical approaches ([9], [14], [19], [20]), the definition of the source term in [13] was obtained by considering a regularization process. This approach led to a rule of interface coupling that was formalized in our preceding works [2] and [3] using the germ terminology introduced in [1].

\footnotetext{
${ }^{1}$ Laboratoire de Mathématiques CNRS UMR 6623, Université de Franche-Comté, 16 route de Gray, 25030 Besançon Cedex, France (boris.andreianov@univ-fcomte.fr)

${ }^{2}$ Laboratoire de Mathématiques, UMR 8628, CNRS-Université Paris-Sud 11, Bât. 425, Faculté des Sciences d'Orsay, Université Paris-Sud 11 F-91405 Orsay CEDEX (frederic.lagoutiere@math.u-psud.fr)

${ }^{3}$ UPMC Univ Paris 06, UMR 7598, LJLL, F-75005, Paris, France (nicolas.seguin@upmc.fr)

${ }^{4}$ CNRS, UMR 7598, LJLL, F-75005, Paris, France

${ }^{5}$ Inria Paris-Rocquencourt, BP 105, F-78153 Le Chesnay Cedex, France

${ }^{6}$ Inria, Villers-lès-Nancy, F-54600, France (takeo.takahashi@inria.fr),

${ }^{7}$ Université de Lorraine, IECL, UMR 7502, Vandoeuvre-lès-Nancy, F-54506, France,

${ }^{8}$ CNRS, IECL, UMR 7502, Vandoeuvre-lès-Nancy, F-54506, France,
} 
Definition 1. The admissibility germ $\mathcal{G}_{\lambda} \subset \mathbb{R}^{2}$ associated with (1) is defined by

$$
\mathcal{G}_{\lambda}=\left\{\left(c_{-}, c_{+}\right) \in \mathbb{R}^{2} ; c_{-}-c_{+}=\lambda\right\} \cup\left\{\left(c_{-}, c_{+}\right) \in \mathbb{R}_{+} \times \mathbb{R}_{-} ; \quad-\lambda \leq c_{-}+c_{+} \leq \lambda\right\} .
$$

In what follows, we use the notation

$$
\mathcal{G}_{\lambda}(V)=(V, V)+\mathcal{G}_{\lambda} \quad(V \in \mathbb{R}) .
$$

The key feature for the subsequent analysis of the problem is the following dissipativity and maximality property of the germ $\mathcal{G}_{\lambda}(V)$ (see [3, Prop. 2.3]):

$$
\left(c_{L}, c_{R}\right) \in \mathcal{G}_{\lambda}(V) \Longleftrightarrow\left[\forall\left(b_{L}, b_{R}\right) \in \mathcal{G}_{\lambda}(V) \Phi\left(c_{L}, b_{L}\right)-V\left|c_{L}-b_{L}\right| \geq \Phi\left(c_{R}, b_{R}\right)-V\left|c_{R}-b_{R}\right|\right] ;
$$

here and in the whole paper, $\Phi$ is the function defined by

$$
\Phi(a, b)=\operatorname{sgn}(a-b)\left(a^{2} / 2-b^{2} / 2\right) \quad(a, b \in \mathbb{R}) .
$$

Now let us give precise definitions of solutions, separately for (1) and for (2). For the sake of simplicity, consider a finite time horizon $T>0$ (global in time solutions are defined via localization to $[0, T]$ for all $T>0$ ).

Definition 2. (i) Given $h \in W^{1, \infty}([0, T] ; \mathbb{R})$, a function $u$ is a solution of (1) with initial datum $u_{0}$ if $u \in L^{\infty}((0, T) \times \mathbb{R}) \cap C\left([0, T] ; L_{\text {loc }}^{1}(\mathbb{R})\right)$, if $u$ is a Kruzhkov entropy solution of the Burgers equation with initial datum $u_{0}$ in $\{(t, x) ; t \in(0, T), x \neq h(t)\}$, and if for a.e. $t \in(0, T)$ the one-sided traces of $u$ at the particle position satisfy

$$
\left(u\left(t, h(t)^{-}\right), u\left(t, h(t)^{+}\right)\right) \in \mathcal{G}_{\lambda}\left(h^{\prime}(t)\right) .
$$

(ii) A function $h$ is a solution of (2) with the initial data $h_{0}, V_{0}$ if $h \in W^{2, \infty}([0, T])$, if $h(0)=h_{0}, h^{\prime}(0)=V_{0}$ and if, given $u$ a Kruzhkov entropy solution of the Burgers equation in $\{(t, x) ; t \in(0, T), x \neq h(t)\}$, we have for a.e. $t \in(0, T)$

$$
m h^{\prime \prime}(t)=\left(\frac{u\left(t, h(t)^{-}\right)^{2}}{2}-h^{\prime}(t) u\left(t, h(t)^{-}\right)\right)-\left(\frac{u\left(t, h(t)^{+}\right)^{2}}{2}-h^{\prime}(t) u\left(t, h(t)^{+}\right)\right) .
$$

(iii) A pair $(u, h)$ satisfying the previous two items is said to be an entropy solution of (1), (2), (3).

Remark 3. The above interpretation (8) of the ODE (2) stems from the principle of conservation of the total momentum $Q(t):=\int_{\mathbb{R}} u(t, \cdot) d x+m h^{\prime}(t)$.

Remark 4. It is worth noticing that, although in the above definition, a Kruzhkov entropy solution $u$ of the Burgers equation is a priori only in $L^{\infty}((0, T) \times \mathbb{R}) \cap C\left([0, T] ; L_{\text {loc }}^{1}(\mathbb{R})\right)$ formulas $(7)$ and (8) make sense: indeed, according to Panov in [17] (see also Kwon, Vasseur [12]), the traces $t \mapsto u\left(t, h(t)^{ \pm}\right)$on the path $\{x=h(t)\}$ exist in the strong $L^{1}$ sense whenever $u$ is an entropy solution away from the path (due to the regularity assumption on the path $h(t))^{1}$.

Yet, the explicit use of traces of $u$ in the above definition makes it delicate to discuss approximation of solutions; fortunately, an equivalent "traceless" formulation can be provided:

Definition 5. (i) Given $h \in W^{1, \infty}([0, T], \mathbb{R})$, a function $u$ is a solution of (1) with initial datum $u_{0}$ if $u \in L^{\infty}((0, T) \times \mathbb{R}) \cap C\left([0, T] ; L_{l o c}^{1}(\mathbb{R})\right)$, if and if for all $\left(\kappa_{L}, \kappa_{R}\right) \in \mathbb{R}^{2}$ there exists $M>0$ such that $\partial_{t}|u-\kappa|+\partial_{x} \Phi(u, \kappa) \leq M \operatorname{dist}_{1}\left(\left(\kappa_{L}, \kappa_{R}\right), \mathcal{G}_{\lambda}\left(h^{\prime}(t)\right)\right) \delta_{0}(x-h(t))$ in $\mathcal{D}^{\prime}\left(\mathbb{R}_{+} \times \mathbb{R}\right)$ with initial datum $u_{0}$, where dist ${ }_{1}$ is the distance on $\mathbb{R}^{2}$ induced by the norm $\|(x, y)\|_{1}=|x|+|y|$, and where

$$
\kappa(t, x):=\kappa_{L} \mathbb{1}_{\mathbb{R}_{-}}(x-h(t))+\kappa_{R} \mathbb{1}_{\mathbb{R}_{+}}(x-h(t)) .
$$

\footnotetext{
${ }^{1}$ In [17], the trace existence result is stated for $C^{1}$ boundary, and it is pointed out that generalization to $W^{1, \infty}$ boundary is straightforward. Also notice that throughout the paper, $W^{1, \infty}$ can be replaced by the space of continuous piecewise $C^{1}$ functions: in this case, the result of [17] can be used piecewise on the interface $\{x=h(t)\}$.
} 
(ii) A function $h$ is a solution of (2) with the initial data $h_{0}, V_{0}$ if $h \in W^{2, \infty}([0, T])$, if $h(0)=h_{0}, h^{\prime}(0)=V_{0}$ and if, given $u$ a Kruzhkov entropy solution of the Burgers equation in $\{(t, x) ; t \in(0, T), x \neq h(t)\}$, for all $\xi \in \mathcal{D}([0, T))$, for all $\psi \in \mathcal{D}(\mathbb{R})$ such that $\psi \equiv 1$ on the set $h([0, T])$, there holds

$$
-m \int_{0}^{T} h^{\prime}(t) \xi^{\prime}(t) d t=m V_{0} \xi(0)+\int_{0}^{T} \int_{\mathbb{R}}\left(u \psi(x) \xi^{\prime}(t)+\frac{u^{2}}{2}(t, x) \xi(t) \psi^{\prime}(x)\right) d x d t+\int_{\mathbb{R}} u_{0}(x) \psi(x) \xi(0) d x .
$$

(iii) A pair $(u, h)$ satisfying the previous two items is said to be an entropy solution of $(1),(2),(3)$.

Proof of the equivalence between Def. 5 et Def. 2. The proof of equivalence of Definition 2(i) and Definition 5(i) follows the guidelines of [3]. Namely, one can perform the change of variable $y=x-h(t)$ in order to rectify the particle path, and then use (5). The equivalence of Definition 2(ii) and Definition 5(ii) comes from an application of the Green-Gauss formula, see [2, Lemma 2.1].

Remark 6. In the above definition, let us stress that we can choose the constant $M$ in (9) such that it depends only on $\left(\kappa_{L}, \kappa_{R}\right)$, and on $\|u\|_{L^{\infty}((0, T) \times \mathbb{R})},\left\|h^{\prime}\right\|_{L^{\infty}(0, T)}$.

We are now in position to state the first main result of this paper:

Theorem 7 ( $L^{\infty}$ case). Assume $u_{0} \in L^{\infty}(\mathbb{R})$ and $h_{0}, V_{0} \in \mathbb{R}$. Then for all $T>0$ there exists at least one solution $(u, h)$ of (1), (2) and (3) in the sense of Definition 2 (or equivalently of Definition 5).

The second main result of this paper is the following

Theorem 8 ( $B V$ case). Assume $u_{0} \in B V(\mathbb{R})$ and $h_{0}, V_{0} \in \mathbb{R}$. Then any solution $(u, h)$ of (1), (2) and (3) in the sense of Definition 2 (or equivalently of Definition 5) satisfies, for all $T>0, u \in L^{\infty}(0, T ; B V(\mathbb{R}))$. Moreover, there exists at most one solution of (1), (2) and (3).

Actually, the uniqueness result of Theorem 8 is a "weak-strong" type uniqueness result (see Theorem 16 for a precise statement). Let us note that the uniqueness in the $L^{\infty}$ case (hypothesis of Theorem 7 ) remains an open problem. Some ingredients of the proof of the theorems are the results of the work [3] of the first and the third authors, where the Burgers equation with singular source term supported by the path $h(t) \equiv 0$ of a stationary particle is considered; it is not difficult to extend them to the case of a general "frozen" particle path (we assume that this path is given and it is $C^{1}$ or piecewise $C^{1}$ ). The other ingredient of Theorem 8 is the new $B V$ estimate that we prove in this paper. These results are presented in Section 2. Using the technique of continuous dependence on the flux of solutions of conservation laws (see Bouchut and Perthame [5], Karlsen and Risebro [10], Lécureux-Mercier [15] and references therein), we evaluate the gap between two solutions of (1) corresponding to different particle paths. Based on this estimate, in Section 3 we derive uniqueness of a solution to the coupled problem (1),(2),(3). Then in Section 4, we describe a splitting approximation scheme for the coupled problem and prove its convergence. Section 5 contains the proof of existence in the $L^{\infty}$ case, that is based upon a fixed-point argument and a tedious check of the continuity of the map that associates the solution $u$ in the sense of Definitions 2(i),5(i) to a given particle path $h$. The Appendix contains the calculations needed to establish the $B V$ estimate; they are based upon the wave-front tracking algorithm of Dafermos [7] as presented in Holden and Risebro [8].

\section{Burgers equation driven by a particle with a given path: known results and new $B V$ estimates}

In this section, we consider a given path $h \in W^{1, \infty}([0, T])$. We study well-posedness for the Cauchy problem for (1) in the sense of Definition 2(i) (or 5(i)).

\subsection{Well-posedness for the Cauchy problem}

With the results and techniques of [3], one can deduce several consequences. Therefore, in the sequel of this subsection, we skip some of the proofs or we only give the main steps.

First, we focus on the case of a continuous and piecewise affine path $h_{\ell}$. Let $0=t^{0}<t^{1}<\cdots<t^{N_{\ell}}=T$ be the sequence of times where the derivative of $h_{\ell}$ is discontinuous and note $\left(V_{\ell}^{n}\right)_{n}$ the associated sequence 
of velocities. Then, we can write $h_{\ell}^{\prime}(t)=\sum_{n=1}^{N_{\ell}} V_{\ell}^{n} \mathbb{1}_{\left(t^{n-1}, t^{n}\right]}(t)$ and we assume without loss of generality that $h_{\ell}(0)=0$. A direct consequence of [3] is the well-posedness result for the Cauchy problem and the following $L^{\infty}$ bound.

Lemma 9. Assume that $u_{0} \in L^{\infty}(\mathbb{R})$ and that $h_{\ell}$ is a given piecewise affine path. Then there exists a unique solution $u_{\ell}$ of (1) in the sense of Definition $2(i)$ (or $5(i)$ ). This solution obeys the bound $\left\|u_{\ell}(t, \cdot)\right\|_{\infty} \leq\left\|u_{0}\right\|_{\infty}+\lambda$.

Proof. The existence and uniqueness proofs were already discussed in [3, Sect.4.2]; our main focus is on the $L^{\infty}$ estimate. Performing the change of variables $v(t, y):=u_{\ell}\left(t, y+V_{\ell}^{1} t\right)-V_{\ell}^{1}$, we notice that in the time interval $\left(0, t^{1}\right], v$ is a solution of

$$
\partial_{t} v+\partial_{y}\left(v^{2} / 2\right)=-\lambda v \delta_{0}, \quad v(0, \cdot)=u_{0}-V_{\ell}^{1}
$$

Let us define

$$
c_{R}:=\min \left\{\operatorname{essinf} u_{\mathbb{R}_{-}} u_{0}-\lambda, \operatorname{essinf} u_{\mathbb{R}_{+}}\right\} \text {and } c_{L}:=c_{R}+\lambda .
$$

Since $\left(c_{L}-V_{\ell}^{1}, c_{R}-V_{\ell}^{1}\right) \in \mathcal{G}_{\lambda}$, the function

$$
c(y):=c_{L} \mathbb{1}_{\mathbb{R}_{-}}(y)+c_{R} \mathbb{1}_{\mathbb{R}_{+}}(y)-V_{\ell}^{1} \quad(y \in \mathbb{R})
$$

is a stationary entropy solution of $\partial_{t} u+\partial_{x}\left(u^{2} / 2\right)=-\lambda u \delta_{0}(x)$ for $t>0$ and $x \in \mathbb{R}$ (see Definition 2, (i), with $h(t)=0$ for any $t)$. Moreover, $v(0, \cdot) \geq c(\cdot)$ and thus, using [3, Thm. 2.6] we deduce that $v(t, y) \geq c(y)$ for all $y \in \mathbb{R}$ and all relevant $t$. Consequently,

$$
u_{\ell}(t, x) \geq c_{L} \mathbb{1}_{\mathbb{R}_{-}}\left(x-h_{\ell}(t)\right)+c_{R} \mathbb{1}_{\mathbb{R}_{+}}\left(x-h_{\ell}(t)\right) \quad\left(x \in \mathbb{R}, t \in\left(0, t^{1}\right]\right) .
$$

By induction, we can prove that for all $n$,

$$
u_{\ell}(t, x) \geq c_{L} \mathbb{1}_{\mathbb{R}_{-}}\left(x-h_{\ell}(t)\right)+c_{R} \mathbb{1}_{\mathbb{R}_{+}}\left(x-h_{\ell}(t)\right) \quad\left(x \in \mathbb{R}, t \in\left(t^{n-1}, t^{n}\right]\right) .
$$

More precisely, we perform the change of variables $v(t, y):=u_{\ell}\left(t+t^{n}, y+h_{\ell}\left(t^{n}\right)+V_{\ell}^{n+1} t\right)-V_{\ell}^{n+1}$ for $t \in\left(t^{n-1}, t^{n}\right]$ and we follow the above proof. Using the same technique to get the upper bound, we finally get

$$
\min \left\{\operatorname{ess} \inf _{\mathbb{R}^{-}} u_{0}-\lambda, \operatorname{ess} \inf _{\mathbb{R}_{+}} u_{0}\right\} \leq u_{\ell}(t, x) \leq \max \left\{\underset{\mathbb{R}^{-}}{\operatorname{ess} \sup _{0}} u_{0}, \operatorname{ess} \sup _{\mathbb{R}_{+}} u_{0}+\lambda\right\} .
$$

Hence the claim follows.

Let us turn now to the case of a particle path $h \in W^{1, \infty}([0, T])$. The uniqueness result can be adapted from [3] (it is actually independent of the previous lemma):

Proposition 10. Assume that $u_{0} \in L^{\infty}(\mathbb{R})$ and that $h \in W^{1, \infty}([0, T])$ is given. There is at most one solution $u$ of equation (1) in the sense of Definition $2(i)$ (or $5(i)$ ).

Proof. We take a sequence of classical test functions $\left(\psi_{n}^{R}\right)_{n \in \mathbb{N}}$ (see [11]) approximating (as $n$ tends to $+\infty$ ) the characteristic function of the trapezoid $\{(t, x) ;|x| \leq R+L(T-t), 0 \leq t \leq T\}$ with $L \geq\|u\|_{\infty}$ and we multiply them by a truncation function $\xi_{n} \in \mathcal{D}\left([0, T] \times \mathbb{R}^{*}\right)$ around the particle path approximating $(t, x) \mapsto \min \{n|x-h(t)|, 1\}$.

Let us consider $u, \hat{u}$ two entropy solutions in the sense of Definition $2(\mathrm{i})$ with the same initial condition $u_{0} \in L^{\infty}(\mathbb{R})$. Using the Kruzhkov doubling of variables method with the test functions $\psi_{n}^{R} \xi_{n}$, which vanish on $\{x=h(t)\}$, we get the so-called Kato inequality

$$
\begin{aligned}
-\int_{0}^{T} \int_{\mathbb{R}}|u-\hat{u}|\left(\partial_{t} \psi_{n}^{R}\right) \xi_{n} d x d t-\int_{0}^{T} \int_{\mathbb{R}} \Phi(u, \hat{u}) & \left(\partial_{x} \psi_{n}^{R}\right) \xi_{n} d x d t \\
& \leq \int_{0}^{T} \int_{\mathbb{R}}(|u-\hat{u}|, \Phi(u, \hat{u})) \cdot\left(\partial_{t} \xi_{n}, \partial_{x} \xi_{n}\right) \psi_{n}^{R} d x d t=: I_{n}
\end{aligned}
$$

where $\cdot$ denotes the scalar product in $\mathbb{R}^{2}$; now, showing that the limit, as $n \rightarrow \infty$, of the right-hand side $I_{n}$ is non-positive, taking $R$ as large as desired, one concludes to the uniqueness of a solution, as in [11]. To study the sign of $\lim _{n \rightarrow \infty} I_{n}$, we introduce the notation

$$
\bar{\Phi}\left(h^{\prime}(t) ; u, \hat{u}\right):=\Phi(u, \hat{u})-h^{\prime}(t)|u-\hat{u}| .
$$


The right-hand side in (12) is an approximation of $\int_{0}^{T} \int_{\mathbb{R}} n \operatorname{sign}(x-h(t)) \bar{\Phi}\left(h^{\prime}(t) ; u, \hat{u}\right) \mathbb{1}_{\left[0, \frac{1}{n}\right]}(|x-h(t)|) \psi_{n}^{R}$, so that using the strong traces of $u$ at $x=h(t)^{ \pm}$at the limit $n \rightarrow \infty$ we can write this term as

$$
-\int_{0}^{T}\left(\bar{\Phi}\left(h^{\prime}(t) ; u\left(t, h(t)^{-}\right), \hat{u}\left(t, h(t)^{-}\right)\right)-\bar{\Phi}\left(h^{\prime}(t) ; u\left(t, h(t)^{+}\right), \hat{u}\left(t, h(t)^{+}\right)\right)\right) \psi_{n}^{R}(h(t)) d t .
$$

Due to the trace condition in Definition 2(i) and to the germ dissipativity property (5), the above term is non-positive. Then, the non-positivity of the left-hand side of (12) implies the uniqueness, following classical arguments. Details can be found in [3], for the particular case $h(\cdot) \equiv 0$.

Using Proposition 10 and Lemma 9, we deduce the following result.

Theorem 11. Assume $h \in W^{1, \infty}([0, T])$ and $u_{0} \in L^{\infty}(\mathbb{R})$, there exists a unique solution $u$ of equation $(1)$ in the sense of Definition 2(i) (or 5(i)). Moreover,

$$
\forall t \in(0, T) \quad\|u(t, \cdot)\|_{\infty} \leq\left\|u_{0}\right\|_{\infty}+\lambda .
$$

Proof. The uniqueness is proved in Proposition 10. In order to prove existence of a solution to (1) we can approximate in $W^{1,1}([0, T])$ the function $h$ by a sequence $\left(h_{\ell}\right)_{\ell}$ of continuous piecewise affine paths. Applying Lemma 9 , we deduce that for each $\ell$, there exists a unique solution $u_{\ell}$ of (1) with initial datum $u_{0} \in L^{\infty}(\mathbb{R})$ and with path $h_{\ell}$. Moreover, the following estimate holds:

$$
\left\|u_{\ell}(t, \cdot)\right\|_{\infty} \leq\left\|u_{0}\right\|_{\infty}+\lambda
$$

Thus we can use the strong compactness result of [18], [16] to deduce that $u_{\ell} \rightarrow u$ a.e. $(0, T) \times \mathbb{R}$. To deduce that $u$ is solution of (1), we write for each $\ell$ the global integral formulation of Definition 5 (i) (it is done in Section 4.2 for the coupled problem) with a positive $\psi \in \mathcal{D}((0, T) \times \mathbb{R})$ : for all $\left(\kappa_{L}, \kappa_{R}\right) \in \mathbb{R}^{2}$ there exists $M>0$ such that

$$
\int_{0}^{T} \int_{\mathbb{R}}\left|u_{\ell}-\kappa\right|\left(\partial_{t} \psi\right) d x d t+\int_{0}^{T} \int_{\mathbb{R}} \Phi\left(u_{\ell}, \kappa\right)\left(\partial_{x} \psi\right) d x d t+M \int_{0}^{T} \operatorname{dist}_{1}\left(\left(\kappa_{L}, \kappa_{R}\right), \mathcal{G}_{\lambda}\left(h_{\ell}^{\prime}(t)\right) \psi\left(h_{\ell}(t)\right) d t \geq 0 .\right.
$$

Note that the constant $M$ above does not depend on $\ell$ since we have the uniform estimate (14) and since $\left\|h_{\ell}^{\prime}\right\|_{\infty} \leq\left\|h^{\prime}\right\|_{\infty}$ (see Remark 6). The result of weak compactness allows to pass to the limit in the first two integrals. For the last one, it is sufficient to notice that for almost every $t \in(0, T)$,

$$
\begin{gathered}
\operatorname{dist}_{1}\left(\left(\kappa_{L}, \kappa_{R}\right), \mathcal{G}_{\lambda}\left(h_{\ell}^{\prime}(t)\right)\right) \leq 2\left|h_{\ell}^{\prime}(t)-h^{\prime}(t)\right|+\operatorname{dist}_{1}\left(\left(\kappa_{L}, \kappa_{R}\right), \mathcal{G}_{\lambda}\left(h^{\prime}(t)\right)\right), \\
\left|\psi\left(h_{\ell}(t)\right)-\psi(h(t))\right| \leq C\left\|h_{\ell}-h\right\|_{\infty} .
\end{gathered}
$$

In particular, since $h_{\ell} \rightarrow h$ in $W^{1,1}(0, T)$, we can pass to the limit in (15) and deduce that $u:=\lim _{N_{\ell} \rightarrow \infty} u_{\ell} \in$ $L^{\infty}((0, T) \times \mathbb{R})$ satisfies the global integral formulation of Definition $5(\mathrm{i})$. To end the proof, we invoke a classical result (see [6]) to deduce that $u \in C\left([0, T] ; L_{l o c}^{1}(\mathbb{R})\right)$.

Remark 12. Notice that another way to prove the convergence of $\left(u_{\ell}\right)_{\ell}$ is to use the continuity estimate (23) below, first with $u_{0} \in B V(\mathbb{R})$ and different paths $h_{\ell}$, then with the fixed path $h$ and a sequence of $B V$ initial data approximating a general $L^{\infty}$ initial datum $u_{0}$.

\subsection{Accurate $B V$ estimates on the solutions}

This paragraph is devoted to careful estimation of the space variation for the unique entropy solution for (1) with $h \equiv 0$. More precisely, we re-consider the following particular case that was the object of [3]:

$$
\partial_{t} u+\partial_{x}\left(u^{2} / 2\right)=-\lambda u \delta_{0}(x) \quad(t>0, x \in \mathbb{R}),
$$

with the initial condition

$$
u(0, x)=u_{0}(x) \quad(x \in \mathbb{R}) .
$$

Even in that case, the problem of existence, in the sense of Definitions 2(i), 5 (i) remains delicate. Specifically, in order to get fine estimates on the solutions, wave interactions with the source path have to be handled carefully. While existence was shown in [3], the method employed was not based on a uniform $B V$ control of approximate solutions ( $B V_{l o c}$ control away from the interface was used instead). Here, constructing the solutions of (16), (17) by the wave-front tracking algorithm (see [8]), we control wave interactions quite precisely and justify the following bound. 
Proposition 13. Assume $u_{0} \in B V(\mathbb{R})$. The unique solution of (16), (17) in the sense of Definitions 2(i) (or $5(i))$ belongs to $L^{\infty}([0, T] ; B V(\mathbb{R}))$ and satisfies

$$
\sup _{t \in(0, T]} \operatorname{Tot} \operatorname{Var} u(t, \cdot) \leq \operatorname{Tot} \operatorname{Var} u_{0}+2 \operatorname{dist}_{1}\left(\left(u_{0}\left(0^{-}\right), u_{0}\left(0^{+}\right)\right), \mathcal{G}_{\lambda}\right) .
$$

A proof of this proposition, based on a wave front tracking, lies in the Appendix. Notice that for approximations $u^{\delta}$ obtained with the wave-front tracking algorithm, we obtain an additional remarkable property: namely, $\operatorname{Tot} \operatorname{Var} u^{\delta}$ is a non-decreasing function of $t \in(0, T]$. At the present stage, we are unable to justify this property for $u=\lim _{\delta \rightarrow 0} u^{\delta}$ solution of (16),(17).

As a consequence, we can improve the result of Theorem 11, giving a precise $B V$ control of solutions corresponding to $B V$ data. In order to state this result we introduce the space

$$
P B V([0, T]):=\left\{h \in W^{1, \infty}([0, T]) ; h^{\prime} \in B V([0, T])\right\} .
$$

Corollary 14. Let $u$ be the solution of (1) with given $h \in P B V([0, T])$ and with initial datum $u_{0} \in B V(\mathbb{R})$. Then for all $t \in(0, T)$ we have

$$
\operatorname{Tot} \operatorname{Var} u(t, \cdot) \leq \operatorname{Tot} \operatorname{Var} u_{0}+2 \operatorname{dist}_{1}\left(\left(u_{0}\left(0^{-}\right), u_{0}\left(0^{+}\right)\right), \mathcal{G}_{\lambda}\left(h^{\prime}(0)\right)\right)+4 \operatorname{Tot}_{\operatorname{Var}_{[0, t]}} h^{\prime} .
$$

Proof. It is easy to see that Proposition 13 can be extended to the case of affine path $h$ by using a change of variable (the important property being $h^{\prime \prime}=0$ ). We choose a sequence of piecewise affine functions $h_{\ell}$ with variation on $[0, T]$ not exceeding the variation of $h^{\prime}(\cdot)$ on $[0, T]$. It is enough to apply the estimate of Proposition 13(ii) on each interval $\left[t_{\ell}^{n-1}, t_{\ell}^{n}\right]$ where the approximate piecewise affine path $h_{\ell}$ is straight. At the initial time, the total variation of $u_{0}$ may increase by $2 \operatorname{dist}_{1}\left(\left(u_{0}\left(0^{-}\right), u_{0}\left(0^{+}\right)\right), \mathcal{G}_{\lambda}\left(h^{\prime}(0)\right)\right)$ due to the interaction that replaces $\left(u_{0}\left(0^{-}\right), u_{0}\left(0^{+}\right)\right)$by a state $\left(c_{L}, c_{R}\right)$ at the interface arising from the solution of the corresponding Riemann problem. At every subsequent step $t=\left(t_{\ell}^{n}\right)^{-}$the pair of traces belongs to $\mathcal{G}_{\lambda}\left(h_{\ell}^{\prime}\left(\left(t_{\ell}^{n}\right)^{-}\right)\right)$and the first interaction replaces it by a pair of states in $\mathcal{G}_{\lambda}\left(h_{\ell}^{\prime}\left(\left(t_{\ell}^{n}\right)^{+}\right)\right)$, therefore, following Proposition 13, we have

$$
\operatorname{Tot} \operatorname{Var} u_{\ell}\left(\left(t_{\ell}^{n}, \cdot\right)^{+}\right) \leq \operatorname{Tot} \operatorname{Var} u_{\ell}\left(\left(t_{\ell}^{n}\right)^{-}, \cdot\right)+2 \operatorname{dist}_{1}\left(\mathcal{G}_{\lambda}\left(h_{\ell}^{\prime}\left(\left(t_{\ell}^{n}\right)^{-}\right)\right), \mathcal{G}_{\lambda}\left(h_{\ell}^{\prime}\left(\left(t_{\ell}^{n}\right)^{+}\right)\right)\right) .
$$

Hence, recalling that $\mathcal{G}_{\lambda}(V)=\mathcal{G}_{\lambda}+(V, V)$, we have

$$
\operatorname{dist}_{1}\left(\mathcal{G}_{\lambda}\left(h_{\ell}^{\prime}\left(\left(t_{\ell}^{n}\right)^{-}\right)\right), \mathcal{G}_{\lambda}\left(h_{\ell}^{\prime}\left(\left(t_{\ell}^{n}\right)^{+}\right)\right)\right) \leq 2\left|h_{\ell}^{\prime}\left(\left(t_{\ell}^{n}\right)^{+}\right)-h_{\ell}^{\prime}\left(\left(t_{\ell}^{n}\right)^{-}\right)\right|
$$

and we deduce

$$
\operatorname{Tot} \operatorname{Var} u_{\ell}(t, \cdot) \leq \operatorname{Tot} \operatorname{Var} u_{0}+2 \operatorname{dist}_{1}\left(\left(u_{0}\left(0^{-}\right), u_{0}\left(0^{+}\right)\right), \mathcal{G}_{\lambda}\left(h^{\prime}(0)\right)\right)+4 \operatorname{Tot} \operatorname{Var}_{[0, t]} h_{\ell}^{\prime}
$$

which yields (19) passing to the limit as in the proof of Theorem 11.

\subsection{Lipschitz continuous dependence of $B V$ solutions with respect to $h$}

Assume $h \in W^{1, \infty}([0, T])$ and $u_{0} \in L^{\infty}(\mathbb{R})$, and consider the unique solution $u$ of equation (1) in the sense of Definition 2(i) (or 5(i)) (see Theorem 11). We make the coordinate transformations that rectify the path of the particle, and consider $w(t, x)=u(t, x+h(t))$. Then $w \in L^{\infty}((0, T) \times \mathbb{R}) \cap C\left([0, T] ; L_{l o c}^{1}(\mathbb{R})\right)$ is the entropy solution of the problem

$$
\begin{gathered}
\partial_{t} w+\partial_{x}\left(w^{2} / 2-V w\right)=\lambda(V-u) \delta_{0}(x) \quad(t \in(0, T), x \in \mathbb{R}), \\
w(0, x)=w_{0}(x) \quad(x \in \mathbb{R}),
\end{gathered}
$$

where $V=h^{\prime} \in L^{\infty}(0, T)$. In particular, setting $\bar{\Phi}(V ; a, b):=|a-b|\left(\frac{a+b}{2}-V\right)$, we deduce from Definition 2 that for all $k \in \mathbb{R}$

$$
\partial_{t}|w-k|+\partial_{x} \bar{\Phi}(V ; w, k) \leq 0 \text { in } \mathcal{D}^{\prime}\left((0, T) \times \mathbb{R}^{*}\right)
$$

(where $\mathbb{R}^{*}$ stands for $\mathbb{R} \backslash\{0\}$ ) and

$$
\left(w\left(t, 0^{-}\right), w\left(t, 0^{+}\right)\right) \in \mathcal{G}_{\lambda}(V(t)) \text { for a.e. } t \in(0, T) .
$$


In this section, we compare two entropy solutions $w$ and $\hat{w}$ of $(20)-(21)$ for two different particle velocities $V$ and $\hat{V}$ and two different initial conditions $w_{0}$ and $\hat{w}_{0}$. The corresponding result applies in the case where at least one of the two solutions enjoys a uniform in time $B V$ in space estimate. Its proof is similar to the arguments of [5] and [10].

Lemma 15. Assume $V, \hat{V} \in L^{\infty}(0, T), w_{0}, \hat{w}_{0} \in L^{\infty}(\mathbb{R})$ and $w_{0}-\hat{w}_{0} \in L^{1}(\mathbb{R})$. Let $w, \hat{w} \in L^{\infty}((0, T) \times \mathbb{R}) \cap$ $C\left([0, T] ; L_{\text {loc }}^{1}(\mathbb{R})\right)$ be the entropy solutions of system $(20)-(21)$ associated respectively with $\left(V, w_{0}\right)$ and $\left(\hat{V}, \hat{w}_{0}\right)$. Assume that there exists $C_{T}$ such that for all $t \in(0, T)$, TotVar $w(t, \cdot) \leq C_{T}$. Then there exists $C \in \mathbb{R}$ such that for all $t<T$

$$
\int_{\mathbb{R}}|\hat{w}-w|(t, x) d x \leq \int_{\mathbb{R}}\left|\hat{w}_{0}-w_{0}\right| d x+\left(C_{T}+C\right) \int_{0}^{t}|\hat{V}-V|(s) d s \in \overline{\mathbb{R}}
$$

$\left(C\right.$ depends on $\|w\|_{\infty},\|\hat{w}\|_{\infty}$ and $\left.\|\hat{V}-V\|_{\infty}\right)$.

Proof. We notice that for all $k \in \mathbb{R}$

$$
\left|\partial_{x}(\bar{\Phi}(\hat{V} ; w, k)-\bar{\Phi}(V ; w, k))\right|=\left|(V-\hat{V}) \partial_{x}\right| w-k|| \leq|V-\hat{V}|\left|\partial_{x} w\right| .
$$

Using (22), we see that $w$ satisfies

$$
\partial_{t}|w-k|+\partial_{x} \bar{\Phi}(\hat{V} ; w, k) \leq \partial_{x}(\bar{\Phi}(\hat{V} ; w, k)-\bar{\Phi}(V ; w, k)) \leq \mu \text { in } \mathcal{D}^{\prime}\left((0, T) \times \mathbb{R}^{*}\right)
$$

where $\mu \in L^{\infty}\left(0, T ; \mathcal{M}_{b}(\mathbb{R})\right)$ is a non-negative measure with

$$
\|\mu\|_{L^{1}\left(0, t ; \mathcal{M}_{b}(\mathbb{R})\right)} \leq C_{T} \int_{0}^{t}|\hat{V}-V| d s
$$

Now we can use (22) and (24) perform the doubling of variables of Kruzhkov, with a test function $\psi_{n}^{R} \xi_{n}$ with, as in the proof of Proposition 10, $\psi_{n}^{R}$ approximating the characteristic function of the trapezoid $\{(t, x) ;|x| \leq$ $R+L(T-t), 0 \leq t \leq T\}$ with $L \geq\|w\|_{\infty}+\|\hat{w}\|_{\infty}+\|V\|_{\infty}$ and $\xi_{n} \in \mathcal{D}\left(\mathbb{R}^{*}\right)$ approximating $x \mapsto \min \{n|x|, 1\}$. Using (25), we deduce the following Kato inequality for all $t \in(0, T)$

$$
\begin{aligned}
\int_{\mathbb{R}}|\hat{w}-w|(t, \cdot) \psi_{n}^{R}(t, \cdot) \xi_{n} d x \leq \int_{0}^{t} \int_{\mathbb{R}} \bar{\Phi}(\hat{V} ; \hat{w}, w) \partial_{x}\left(\psi_{n}^{R} \xi_{n}\right) d x d s & +\int_{0}^{t} \int_{\mathbb{R}}|\hat{w}-w| \partial_{t} \psi_{n}^{R} \xi_{n} d x d s \\
& +\int_{\mathbb{R}}\left|\hat{w}_{0}-w_{0}\right| \psi_{n}^{R}(0, \cdot) \xi_{n} d x+\left\|\psi_{n}^{R} \xi_{n}\right\|_{\infty} C_{T} \int_{0}^{t}|\hat{V}-V| d s
\end{aligned}
$$

Using the properties of $\bar{\Phi}(\hat{V} ; \hat{w}, w)$ with the choice of $L$, we deduce

$$
\begin{aligned}
\int_{\mathbb{R}}|\hat{w}-w|(t, \cdot) \psi_{n}^{R}(t, \cdot) \xi_{n} d x \leq \int_{0}^{t} \int_{\mathbb{R}} \bar{\Phi}(\hat{V} ; \hat{w}, w) \psi_{n}^{R} \partial_{x} \xi_{n} d x d s & \\
& +\int_{\mathbb{R}}\left|\hat{w}_{0}-w_{0}\right| \psi_{n}^{R}(0, \cdot) \xi_{n} d x+C_{T} \int_{0}^{t}|\hat{V}-V| d s .
\end{aligned}
$$

Proceeding as in the proof of Theorem 11, taking $n \rightarrow \infty$, we find

$$
\begin{aligned}
\int_{\mathbb{R}}|\hat{w}-w|(t, \cdot) \psi_{n}^{R}(t, \cdot) d x \leq \int_{\mathbb{R}} \mid \hat{w}_{0} & -w_{0}\left|\psi_{n}^{R}(0, \cdot) d x+C_{T} \int_{0}^{t}\right| \hat{V}-V \mid d s \\
& +\int_{0}^{t}\left(\bar{\Phi}\left(\hat{V}(t) ; \hat{w}\left(t, 0^{+}\right), w\left(t, 0^{+}\right)\right)-\bar{\Phi}\left(\hat{V}(t) ; \hat{w}\left(t, 0^{-}\right), w\left(t, 0^{-}\right)\right)\right) d s .
\end{aligned}
$$

It remains to estimate the last term of the above inequality. In order to do this, we recall that, for a.e. $t>0$,

$$
\left(w\left(t, 0^{-}\right), w\left(t, 0^{+}\right)\right) \in \mathcal{G}_{\lambda}(V(t))=\mathcal{G}_{\lambda}(\hat{V}(t))-(\Delta(t), \Delta(t)), \quad\left(\hat{w}\left(t, 0^{-}\right), \hat{w}\left(t, 0^{+}\right)\right) \in \mathcal{G}_{\lambda}(\hat{V}(t))
$$


with $\Delta:=\hat{V}-V$. This suggests the following decomposition (removing the time variable for readability):

$$
\begin{aligned}
\left(\bar{\Phi}\left(\hat{V} ; \hat{w}\left(0^{+}\right), w\left(0^{+}\right)\right)-\bar{\Phi}\left(\hat{V} ; \hat{w}\left(0^{-}\right), w\left(0^{-}\right)\right)\right)= & \left(\bar{\Phi}\left(\hat{V} ; \hat{w}\left(0^{+}\right), w\left(0^{+}\right)\right)-\bar{\Phi}\left(\hat{V} ; \hat{w}\left(0^{+}\right), w\left(0^{+}\right)+\Delta\right)\right) \\
+ & +\left(\bar{\Phi}\left(\hat{V} ; \hat{w}\left(0^{+}\right), w\left(0^{+}\right)+\Delta\right)-\bar{\Phi}\left(\hat{V} ; \hat{w}\left(0^{-}\right), w\left(0^{-}\right)+\Delta\right)\right) \\
& +\left(\bar{\Phi}\left(\hat{V} ; \hat{w}\left(0^{-}\right), w\left(0^{-}\right)+\Delta\right)-\bar{\Phi}\left(\hat{V} ; \hat{w}\left(0^{-}\right), w\left(0^{-}\right)\right)\right) \\
=: & E_{r}+E_{o}+E_{l}
\end{aligned}
$$

respectively. By the dissipativity property (5) of the germs and from (29), we deduce $E_{o} \leq 0$ (because $\left.\left(w\left(0^{-}\right)+\Delta, w\left(0^{+}\right)+\Delta\right) \in \mathcal{G}_{\lambda}(\hat{V}(t))\right)$. Moreover, using that $\bar{\Phi}$ is a locally Lipschitz continuous function, we obtain

$$
\left|E_{l}\right|+\left|E_{r}\right| \leq|\Delta| C\left(\|w\|_{\infty},\|\hat{w}\|_{\infty},\|\hat{V}\|_{\infty}\right)
$$

for $\Delta$ sufficiently small, where $C$ is the (local) Lipschitz modulus of $\bar{\Phi}$. Therefore

$$
\left(\bar{\Phi}\left(\hat{V} ; \hat{w}\left(0^{+}\right), w\left(0^{+}\right)\right)-\bar{\Phi}\left(\hat{V} ; \hat{w}\left(0^{-}\right), w\left(0^{-}\right)\right)\right) \leq|V-\hat{V}| C\left(\|w\|_{\infty},\|\hat{w}\|_{\infty},\|\hat{V}\|_{\infty}\right) .
$$

Combining the above estimate with (28) and taking $R \rightarrow \infty$, we conclude the proof of the lemma.

\section{Uniqueness for the coupled problem with $B V$ initial data}

Based on the result of Section 2.3 and on the $B V$ estimate of Section 2.2, we are able to prove the uniqueness of an entropy solution to the Burgers equation driven by a particle with a given path in $P B V([0, T])$, in the sense of Definition 2 (iii) (or equivalently Definition 5 (iii)).

Theorem 16. Assume $u_{0} \in B V(\mathbb{R})$ and assume there exists a solution $(u, h)$ of problem (1),(2),(3) in the sense of Definition 2 and such that $u \in L^{\infty}(0, T ; B V(\mathbb{R}))$. Then this solution is unique within the whole class of (not necessarily $B V$ in space) solutions of (1),(2),(3).

Proof. Assume that $(u, h)$ is solution of $(1),(2),(3)$ in the sense of Definition 2 with $u \in L^{\infty}(0, T ; B V(\mathbb{R}))$. We consider $C_{T}$ such that $\operatorname{Tot} \operatorname{Var} u(t, \cdot) \leq C_{T}$ for almost every $t \in[0, T]$. Assume that

$$
(\hat{u}, \hat{h}) \in\left[L^{\infty}((0, T) \times \mathbb{R}) \cap C\left([0, T] ; L_{l o c}^{1}(\mathbb{R})\right)\right] \times W^{1, \infty}([0, T])
$$

is another solution with the same initial data. Making the change of variables of Section 2.3

$$
w(t, y)=u(t, y+h(t)), \quad \hat{w}(t, y)=\hat{u}(t, y+\hat{h}(t)) .
$$

we find for all $t<T$

$$
\begin{aligned}
\int_{\mathbb{R}}|\hat{u}(t, x)-u(t, x)| d x=\int_{\mathbb{R}} \mid \hat{u}(t, x+\hat{h}(t))-u(t, & x+\hat{h}(t)) \mid d x \\
& \leq \int_{\mathbb{R}}|\hat{w}(t, y)-w(t, y)| d y+|h(t)-\hat{h}(t)| \operatorname{Tot} \operatorname{Var} u(t, \cdot),
\end{aligned}
$$

and finally thanks to Lemma 15 we have

$$
\int_{\mathbb{R}}|\hat{u}(t, x)-u(t, x)| d x \leq\left(2 C_{T}+C\left(\|u\|_{\infty},\|\hat{u}\|_{\infty},\left\|h^{\prime}-\hat{h}^{\prime}\right\|_{\infty}\right)\right) \int_{0}^{t}\left|h^{\prime}(s)-\hat{h}^{\prime}(s)\right| d s,
$$

where we have bounded $|h(t)-\hat{h}(t)|$ by $\int_{0}^{t}\left|h^{\prime}(s)-\hat{h}^{\prime}(s)\right| d s$ by using the fact that $h(0)=\hat{h}(0)$.

Now, we use formulation (10) to estimate the right hand side of (30). Taking $\psi$ suitable for both paths $h$ and $\hat{h}$ and using an approximation of $\xi=\mathbb{1}_{[0, s]}$, we obtain

$$
m\left(h^{\prime}(s)-h^{\prime}(0)\right)=-\int_{\mathbb{R}} u(s, \cdot) \psi+\int_{\mathbb{R}} u_{0} \psi+m h^{\prime}(0) \xi(0)+\int_{0}^{s} \int_{\mathbb{R}} \frac{u^{2}}{2} \psi^{\prime}+\int_{\mathbb{R}} u_{0} \psi \xi(0),
$$

and a similar formula for $\hat{h}^{\prime}(s)$. Making the difference of the two expressions yields

$$
m\left|h^{\prime}(s)-\hat{h}^{\prime}(s)\right| \leq C\left(\|u\|_{\infty},\|\hat{u}\|_{\infty}\right)\left(\int_{\mathbb{R}}|u(s, x)-\hat{u}(s, x)| d x+\int_{0}^{s}\|u(\tau, \cdot)-\hat{u}(\tau, \cdot)\|_{L^{1}(\mathbb{R})} d \tau\right)
$$

(actually $C$ may depend on $\psi$ ). Inserting this estimate in (30) and using the Gronwall inequality allows to conclude $(\hat{u}, \hat{h})=(u, h)$. 


\section{A splitting scheme and existence for the coupled problem}

In this section, starting from $B V$ data $u_{0}$ we construct approximate solutions and prove their convergence to a solution of the coupled problem; together with the uniqueness result of the previous section, this establishes Theorem 8.

\subsection{Splitting algorithm}

Take $N \in \mathbb{N}^{*}=\mathbb{N} \backslash\{0\}$ and set $\Delta t=T / N>0$. We set $t_{n}^{N}=n \Delta t$ for $n \leq N$ and we define the approximate solutions $\left(u^{N}, h^{N}\right)$ by the following algorithm.

- Define $u_{0}^{N}:=u_{0} \in B V(\mathbb{R}), h_{0}^{N}=h_{0}$ and $V_{0}^{N}=V_{0}$.

- Assume that we know $u_{n}^{N}, h_{n}^{N}$ and $V_{n}^{N}$.

To compute $u_{n+1}^{N}, h_{n+1}^{N}$ and $V_{n+1}^{N}$, we solve the following two steps.

1. we define $h^{N}$ on $\left(t_{n}^{N}, t_{n+1}^{N}\right]$ by

$$
h^{N}(t)=h_{n}^{N}+V_{n}^{N}\left(t-t_{n}^{N}\right),
$$

and $u^{N}$ on $\left(t_{n}^{N}, t_{n+1}^{N}\right]$ as the solution of the problem

$$
\partial_{t} u+\partial_{x}\left(u^{2} / 2\right)=-\lambda\left(u-V_{n}^{N}\right) \delta_{0}\left(x-h^{N}(t)\right),\left.\quad u\right|_{t=t_{n}^{N}}=u_{n}^{N} ;
$$

in the sense of Definitions 2(i) with the straight path $h^{N}$.

2 . Then, we write $u_{n+1}^{N}:=u^{N}\left(t_{n+1}^{N}\right), h_{n+1}^{N}:=h^{N}\left(t_{n+1}^{N}\right)$, and

$$
V_{n+1}^{N}:=V_{n}^{N}-\frac{1}{m} \int_{t_{n}^{N}}^{t_{n+1}^{N}} \llbracket \frac{\left(u^{N}(t)\right)^{2}}{2}-V_{n}^{N} u^{N}(t) \rrbracket_{x=h^{N}(t)} d t
$$

where $\llbracket \alpha(t) \rrbracket=\alpha\left(t, h^{N}(t)^{+}\right)-\alpha\left(t, h^{N}(t)^{-}\right)$denotes the jump across the path $h^{N}$ of the quantity $\alpha(t)$.

Remark 17. In the spirit of (10), fixing some function $\psi \in \mathcal{D}(\mathbb{R})$ satisfying $\psi \equiv 1$ on the subset $h^{N}([0, T])$ of $\mathbb{R}$ and $\xi(t)=\mathbb{1}_{\left[t_{n}^{N}, t_{n+1}^{N}\right]}(t)$, and using the Green-Gauss formula, we can propose the alternative equation for $V_{n+1}^{N}$ :

$$
m\left(V_{n+1}^{N}-V_{n}^{N}\right)=\left(\int_{t_{n}^{N}}^{t_{n+1}^{N}} \int_{\mathbb{R}} \frac{\left(u^{N}(t)\right)^{2}}{2} \psi^{\prime} d x d t+\int_{\mathbb{R}}\left(u_{n}^{N}-u_{n+1}^{N}\right) \psi d x\right) .
$$

From Theorem 11, it is clear that the algorithm makes sense, that is to say that it yields a unique solution $\left(h^{N}, u^{N}\right)$ defined in $[0, T]$ for any $N \in \mathbb{N}^{*}$. In the next paragraph, we prove compactness and convergence of the splitting approximation procedure as $N \rightarrow+\infty$.

\subsection{Compactness and passage to the limit}

Assume for simplicity that the initial datum $u_{0}$ is compactly supported (the general case follows using the property of finite speed of propagation, see [11]). Notice that in this case, $u^{N} \in L^{\infty}\left(0, T ; L^{1}(\mathbb{R})\right)$.

Using Theorem 11, we obtain that for all $N$, the sequence $\left(u^{N}\right)$ satisfies

$$
\left\|u^{N}\right\|_{\infty} \leq\left\|u_{0}\right\|_{\infty}+\lambda
$$

Using this estimate and (32), we deduce that there exists a positive constant $C$ such that

$$
\left|V_{n+1}^{N}-V_{n}^{N}\right| \leq C \Delta t+C \Delta t\left|V_{n}^{N}\right| \quad \text { and } \quad\left|V_{n+1}^{N}\right| \leq(1+C \Delta t)\left|V_{n}^{N}\right|+C \Delta t
$$

Thanks to a discrete Gronwall lemma, the above estimate yields that $\left(V_{n}^{N}\right)$ is bounded independently of $n \in$ $\{0, \ldots, N\}$ and $N$, and thus that there exists a constant $C$ independent of $N$ such that

$$
\left\|h^{N}\right\|_{\infty}+\left\|V^{N}\right\|_{\infty}+\operatorname{Tot} \operatorname{Var}_{(0, T)} V^{N} \leq C .
$$


Using Helly's theorem, we deduce that (up to a subsequence) $V^{N} \rightarrow V$ in $L^{1}(0, T)$ and that $h^{N} \rightarrow h$ in $W^{1,1}(0, T)$, with $V^{\prime}=h$. Moreover, using Corollary 14, we deduce that there exists a constant $C$ independent of $N$ such that

$$
\operatorname{Tot}_{\operatorname{Var}_{\mathbb{R}}} u^{N} \leq C \quad(t \in(0, T), N \geq 0) .
$$

Setting $w^{N}(t, x)=u^{N}\left(t, x+h^{N}(t)\right)$ and applying Lemma 15, we see that

$$
w^{N} \rightarrow w \quad \text { in } L^{\infty}\left(0, T ; L^{1}(\mathbb{R})\right)
$$

We then set $u(t, x)=w(t, x-h(t))$ and we deduce from the above results that

$$
u \in L^{\infty}\left(0, T ; L^{1}(\mathbb{R}) \cap B V(\mathbb{R})\right) \cap L^{\infty}((0, T) \times \mathbb{R}) .
$$

Proceeding as in the proof of Theorem 16 (see (30)), we deduce that $u^{N} \rightarrow u$ in $L^{\infty}\left(0, T ; L^{1}(\mathbb{R})\right)$ up to a subsequence.

It remains to show that $(u, h)$ is a solution to problem $(1),(2),(3)$. First, we write for each $N$ the global integral formulation of Definition $5(\mathrm{i})$ with a non-negative $\psi \in \mathcal{D}((0, T) \times \mathbb{R})$ : for all $\left(\kappa_{L}, \kappa_{R}\right) \in \mathbb{R}^{2}$ and for all $\kappa$ as in Definition $5(1)$, there exists $M>0$ such that

$$
\int_{0}^{T} \int_{\mathbb{R}}\left|u^{N}-\kappa\right|\left(\partial_{t} \psi\right) d x d t+\int_{0}^{T} \int_{\mathbb{R}} \Phi\left(u^{N}, \kappa\right)\left(\partial_{x} \psi\right) d x d t+M \int_{0}^{T} \operatorname{dist}_{1}\left(\left(\kappa_{L}, \kappa_{R}\right), \mathcal{G}_{\lambda}\left(V^{N}\right)\right) \psi\left(t, h^{N}(t)\right) d t \geq 0
$$

Note that the constant $M$ above does not depend on $N$ because of the above uniform estimates (34) on $u^{N}$ and (35) on $V^{N}$ (see Remark 6). From the convergence of $\left(u^{N}\right)$, we can pass to the limit in the above formulation, taking into account that

$$
\operatorname{dist}_{1}\left(\left(\kappa_{L}, \kappa_{R}\right), \mathcal{G}_{\lambda}\left(V^{N}(t)\right)\right) \leq \operatorname{dist}_{1}\left(\left(\kappa_{L}, \kappa_{R}\right), \mathcal{G}_{\lambda}(V(t))\right)+2\left|V^{N}(t)-V(t)\right| .
$$

Finally, to obtain (10), we first derive its discrete version from (33). To this end, we take $\xi \in \mathcal{D}([0, T))$, we set $\xi_{n}^{N}=\xi\left(t_{n}^{N}\right)$ for $n \in\{0, \ldots, N\}$, and we multiply (33) by $\xi_{n}^{N}$ and sum over $n \geq 0$. The Abel transform yields

$$
-m \sum_{n=0}^{N-1} \Delta t V_{n}^{N} \frac{\xi_{n+1}^{N}-\xi_{n}^{N}}{\Delta t}-m V_{0} \xi_{0}=\sum_{n=0}^{N-1} \xi_{n}^{N} \int_{t_{n}^{N}}^{t_{n+1}^{N}} \int_{\mathbb{R}} \frac{\left(u^{N}\right)^{2}}{2} \partial_{x} \psi+\sum_{n=0}^{N-1} \Delta t \int_{\mathbb{R}} u_{n}^{N} \frac{\xi_{n+1}^{N}-\xi_{n}^{N}}{\Delta t} \psi+\int_{\mathbb{R}} u_{0} \psi \xi_{0} .
$$

Passing to the limit as $N \rightarrow \infty$ gives (10).

Since $h \in P B V([0, T])$ and using that $u$ is regular enough (see (36)), we conclude that $h$ satisfies (8). Consequently, $h \in W^{2, \infty}([0, T])$. This concludes the proof of Theorem 8 .

Note that thanks to the uniqueness Theorem 16 , the whole sequence $\left(u^{N}, h^{N}\right)_{N \in \mathbb{N}^{*}}$ actually converges.

\section{Proof of Theorem 7: existence for $L^{\infty}$ initial data}

The goal of this section is to prove the existence result in $L^{\infty}$ setting, i.e. Theorem 7 . We use the fixed-point theory in the following way. Fix $u_{0} \in L^{\infty}(\mathbb{R}), h_{0}, V_{0} \in \mathbb{R}$, fix $T>0$ and define

$$
E:=\left\{h \in C^{1}([0, T]): h(0)=h_{0}, h^{\prime}(0)=V_{0}\right\} \text { endowed with the } C^{1}([0, T]) \text { norm. }
$$

Then the map $\mathcal{A}$ is defined as follows: let us consider $h \in E$. Then

- We first associate the solution $u$ of (1) in the sense of Definition 2(i) (or 5(i)) which is given by Theorem 11; note in particular that the traces $t \mapsto u\left(t, h(t)^{ \pm}\right)$on the path $\{x=h(t)\}$ exist in the strong $L^{1}$ sense.

- Second, we define by $h^{*} \in W^{2, \infty}([0, T])$ the solution of $h^{*}(0)=h_{0},\left(h^{*}\right)^{\prime}(0)=V_{0}$ and

$$
m\left(h^{*}\right)^{\prime \prime}(t)=\left(\frac{u\left(t, h(t)^{-}\right)^{2}}{2}-h^{\prime}(t) u\left(t, h(t)^{-}\right)\right)-\left(\frac{u\left(t, h(t)^{+}\right)^{2}}{2}-h^{\prime}(t) u\left(t, h(t)^{+}\right)\right),
$$

where $u$ is obtained from $h$ in the first step.

This solution is expressed as $h^{*}=\mathcal{A}(h)$. 
Remark 18. Let us notice that a fixed point $h$ to $\mathcal{A}$ and the corresponding solution $u$ of (1) in the sense of Definition 2(i) (or $5(\mathrm{i})$ ) which is given by Theorem 11 provide a solution $(h, u)$ to our problem.

We notice that an application of the Green-Gauss formula yields that, whenever $u$ solves the Burgers equation away from the path $\{x=h(t)\}$, equation (38) is equivalent to the fact that for all $\xi \in \mathcal{D}([0, T))$, for all $\psi \in \mathcal{D}(\mathbb{R})$ such that $\psi \equiv 1$ on the set $h([0, T])$, there holds

$$
m \int_{0}^{T}\left(h^{*}\right)^{\prime \prime}(t) \xi(t) d t=\int_{0}^{T} \int_{\mathbb{R}}\left(u \psi \xi^{\prime}+\frac{u^{2}}{2} \xi \psi^{\prime}\right) d x d t+\int_{\mathbb{R}} u_{0} \psi \xi(0) d x
$$

(see (10) in Definition 5). Therefore, in the second step of the definition of $\mathcal{A},(38)$ can be replaced by (39).

Theorem 19. The mapping $\mathcal{A}: E \rightarrow E$ is continuous.

Proof. We split the proof into two parts corresponding to the above construction of $\mathcal{A}$. The most delicate part of this proof is to show that the solution $u$ of (1) depends continuously on $h$. Due to the finite speed of propagation property for the Burgers equation and to the boundedness of the set of relevant paths $\hat{h}$, we can assume that $u_{0}$ is an $L^{1} \cap L^{\infty}$ function.

Step 1

Consider $h \in E$ and $u$ the associated solution of (1) with initial datum $u_{0}$ given by Theorem 11 . Fix $\varepsilon>0$.

Given $\delta>0$, consider another path $\hat{h} \in E$ such that $\|h-\hat{h}\|_{E} \leq \delta$; let $\hat{u}$ be the associated solution of (1) (given by Theorem 11). Using the same kind of change of variables as in Sections 2.3 and 4.2 , we set $w(t, x)=u(t, x+h(t)), \hat{w}(t, x)=\hat{u}(t, x+h(t))$ and $\tilde{h}(t)=\hat{h}(t)-h(t)$. Then

$$
\begin{gathered}
w_{0}(x):=w(0, x)=u_{0}\left(x+h_{0}\right), \quad \hat{w}_{0}(x):=\hat{w}(0, x)=u_{0}\left(x+h_{0}\right), \\
\|\tilde{h}\|_{E}=|\tilde{h}(t)|+\left|(\tilde{h})^{\prime}(t)\right| \leq \delta,
\end{gathered}
$$

and $w$ and $\hat{w}$ are Kruzhkov entropy solutions of

$$
\partial_{t} w+\partial_{x}\left(\frac{w^{2}}{2}-h^{\prime}(t) w\right)=0 \text { for } t \in \mathbb{R}_{+}, x \in \mathbb{R}^{*} \quad \text { and } \partial_{t} \hat{w}+\partial_{x}\left(\frac{\hat{w}^{2}}{2}-h^{\prime}(t) \hat{w}\right)=0 \text { for } t \in \mathbb{R}_{+}, x-\tilde{h}(t) \in \mathbb{R}^{*}
$$

with interface coupling prescribed by the germs $\mathcal{G}_{\lambda}\left((\hat{h})^{\prime}(t)\right)-(\Delta, \Delta)$ and $\mathcal{G}_{\lambda}\left((\hat{h})^{\prime}(t)\right)$, respectively, where $\Delta=$ $(\tilde{h})^{\prime}(t)$. Notice that, unlike in Subsection 2.3, we use the same change of variable on the two equations; consequently, we have the same differential operator on the left, but the source terms are localized on two different interfaces.

Fix $T>0$ and introduce the traces $w\left(t, 0^{ \pm}\right)$of $w$ on $\{x=0\}$; in particular,

for all $\eta, \alpha>0$, there exists $r$ such that for all $y \in(0, r)$ meas $\left\{t \in(0, T) ;\left|w(t, \pm y)-w\left(t, 0^{ \pm}\right)\right|>\eta / 2\right\}<\alpha$

because $L^{1}$ convergence implies convergence in measure. Further, we can approximate the measurable vector map $t \in(0, T) \mapsto\left(w\left(t, 0^{-}\right), w\left(t, 0^{+}\right)\right) \in \mathcal{G}_{\lambda}\left(h^{\prime}(t)\right)$ a.e. by a piecewise constant map as follows:

for all $\eta, \alpha>0$, there exist $N$ and $\left(c_{n}^{ \pm}\right)_{n=1}^{N}$ such that meas $\left\{t \in(0, T) ;\left|w\left(t, 0^{ \pm}\right)-C_{N}^{ \pm}(t)\right|>\eta / 2\right\}<\alpha$

where $C_{N}^{ \pm}(t):=\sum_{n=1}^{N} c_{n}^{ \pm} \mathbb{1}_{\left(t_{n-1}, t_{n}\right]}(t)$ and $t_{n}:=n T / N$; since $h^{\prime}$ is continuous, we can pick $\left(c_{n}^{-}, c_{n}^{+}\right) \in \mathcal{G}_{\lambda}\left(h^{\prime}\left(t_{n}\right)\right)$; moreover, we can assume that $C_{N}^{ \pm}$satisfies the same $L^{\infty}$ bound as $w$. Note that both $r$ and $N$ depend on $u$ and thus on $h$, but they are independent of $\delta$.

Now, assuming that $\delta<1 / 4$, let us consider $\psi_{0}^{\delta} \in \mathcal{D}(-\sqrt{\delta}, \sqrt{\delta})$ such that $0 \leq \psi_{0}^{\delta} \leq 1, \psi_{0}^{\delta} \equiv 1$ in $(-2 \delta, 2 \delta)$, $\left\|\partial_{x} \psi_{0}^{\delta}\right\|_{L^{\infty}} \leq \frac{2}{\sqrt{\delta}}$. We set $\psi_{\infty}^{\delta}=1-\psi_{0}^{\delta}$ (defined on $\left.\mathbb{R}\right)$. In particular, $\operatorname{supp}\left(\psi_{\infty}^{\delta}\right) \subset \mathbb{R} \backslash(-2 \delta, 2 \delta)$ and we can write

$$
\int_{\mathbb{R}}|w-\hat{w}|(T, \cdot)=\int_{\mathbb{R}}|w-\hat{w}|(T, \cdot) \psi_{\infty}^{\delta}+\int_{\mathbb{R}}|w-\hat{w}|(T, \cdot) \psi_{0}^{\delta}=: I_{\infty}^{\delta}+I_{0}^{\delta}
$$

Since $u, \hat{u} \in L^{\infty}((0, T) \times \mathbb{R})$,

$$
\left|I_{0}^{\delta}\right| \leq C \sqrt{\delta}
$$

with a constant $C$ that only depends on $T$ and on the $L^{\infty}$ bounds on $u, \hat{u}$. To estimate the first term of (44), notice that by $(41)$ and by construction of $\psi_{\infty}^{\delta}, w$ and $\hat{w}$ satisfy the same conservation law without source in 
$\operatorname{supp} \psi_{\infty}^{\delta}$. Thus we can deal with the first term of (44) by using the Kato inequality with the test function $\psi_{\infty}^{\delta}(y)$ :

$$
I_{\infty}^{\delta} \leq \int_{\mathbb{R}}\left|w_{0}-\hat{w}_{0}\right| \psi_{\infty}^{\delta}+\int_{0}^{T} \int_{\mathbb{R}} \bar{\Phi}\left(h^{\prime}(t) ; w, \hat{w}\right) \partial_{x} \psi_{\infty}^{\delta}=-\int_{0}^{T} \int_{\mathbb{R}} \bar{\Phi}\left(h^{\prime}(t) ; w, \hat{w}\right) \partial_{x} \psi_{0}^{\delta}
$$

(see (40)), where $\bar{\Phi}$ is the Kruzhkov entropy flux $\bar{\Phi}\left(h^{\prime}(t) ; a, b\right):=\frac{(a+b)|a-b|}{2}-h^{\prime}(t)|a-b|$ associated with the hyperbolic operator $\partial_{t} w+\partial_{x}\left(\frac{w^{2}}{2}-h^{\prime}(t) w\right)$.

Then we write

$$
\begin{aligned}
\bar{\Phi}\left(h^{\prime}(t) ; w, \hat{w}\right) & =\bar{\Phi}\left(h^{\prime}(t) ; \hat{C}_{N}, \hat{w}\right)+\left[\bar{\Phi}\left(h^{\prime}(t) ; C_{N}, \hat{w}\right)-\bar{\Phi}\left(h^{\prime}(t) ; \hat{C}_{N}, \hat{w}\right)\right]+\left[\bar{\Phi}\left(h^{\prime}(t) ; w, \hat{w}\right)-\bar{\Phi}\left(h^{\prime}(t) ; C_{N}, \hat{w}\right)\right] \\
=: & \mathcal{T}_{1}+\mathcal{T}_{2}+\mathcal{T}_{3},
\end{aligned}
$$

(respectively) where

$$
\begin{aligned}
C_{N}(t, x) & =\sum_{n=1}^{N}\left(c_{n}^{-} \mathbb{1}_{\mathbb{R}_{-}}(x)+c_{n}^{+} \mathbb{1}_{\mathbb{R}_{+}}(x)\right) \mathbb{1}_{\left(t_{n-1}, t_{n}\right]}(t)=C_{N}^{-}(t) \mathbb{1}_{\mathbb{R}_{-}}(x)+C_{N}^{+}(t) \mathbb{1}_{\mathbb{R}_{+}}(x), \\
\hat{C}_{N}(t, x) & =\sum_{n=1}^{N}\left(c_{n}^{-} \mathbb{1}_{\mathbb{R}_{-}}(x-\tilde{h}(t))+c_{n}^{+} \mathbb{1}_{\mathbb{R}_{+}}(x-\tilde{h}(t))\right) \mathbb{1}_{\left(t_{n-1}, t_{n}\right]}(t) \\
& =C_{N}^{-}(t) \mathbb{1}_{\mathbb{R}_{-}}(x-\tilde{h}(t))+C_{N}^{+}(t) \mathbb{1}_{\mathbb{R}_{+}}(x-\tilde{h}(t))=C_{N}(t, x-\tilde{h}(t)) .
\end{aligned}
$$

Recall that $\bar{\Phi}$ is a locally Lipschitz continuous function and that $w, C_{N}, \hat{C}_{N}$ and $\hat{w}$ enjoy a uniform $L^{\infty}$ bound. Because the functions $C_{N}$ and $\hat{C}_{N}$ only differ on the set $\{(t, x) ; x$ is between 0 and $\tilde{h}(t)\}$ which measure is at most $T \delta$, there exist $A, C$ such that

$$
\left|\int_{0}^{T} \int_{\mathbb{R}} \mathcal{T}_{2} \partial_{x} \psi_{0}^{\delta}\right| \leq \frac{A}{\sqrt{\delta}} \int_{0}^{T} \int_{\mathbb{R}}\left|C_{N}-\hat{C}_{N}\right| \leq C \sqrt{\delta}
$$

As far as $\mathcal{T}_{3}$ is concerned, for $\eta, \alpha>0$, let us choose $\delta<\sqrt{r}$ with $r$ small enough as in (42), and $N$ large enough as in (43). We obtain that there exist $A_{1}, A_{2}$, and $C$ such that

$$
\left|\int_{0}^{T} \int_{\mathbb{R}} \mathcal{T}_{3} \partial_{x} \psi_{0}^{\delta}\right| \leq \frac{A_{1}}{\sqrt{\delta}} \int_{-\sqrt{\delta}}^{\sqrt{\delta}}\left(\int_{0}^{T}\left|w-C_{N}\right|\right) \leq \frac{A_{1}}{\sqrt{\delta}} 2 \sqrt{\delta}\left(A_{2} \alpha+T \eta\right) \leq C(\alpha+\eta)
$$

Here, we have split, for $y \in[-\sqrt{\delta}, \sqrt{\delta}]$, the integral on $[0, T]$ into an integral on the measurable set $\{t \in$ $\left.(0, T) ;\left|w(t, y)-C_{N}(t)\right|>\eta\right\}$ and an integral on its complement in $[0, T]$. We then have used (42) and the fact that $w-C_{N}$ is bounded.

Finally, to estimate the contribution of $\mathcal{T}_{1}$ to the right-hand side of (46) we use the global entropy inequalities for $\hat{w}$ in each interval $\left[t_{n-1}, t_{n}\right]$ (notice that $\hat{C}_{N}$ is constant on each side of the interface $\{y=\tilde{h}(t)\}$, as required in the global entropy inequalities

$$
\partial_{t}|w-\kappa|+\partial_{x} \bar{\Phi}\left(h^{\prime}(t), w, \kappa\right) \leq M \operatorname{dist}_{1}\left(\left(\kappa_{L}, \kappa_{R}\right), \mathcal{G}_{\lambda}\left(h^{\prime}(t)\right)\right) \delta_{0}
$$

which can be deduced from (9). We have, for $n=1, \ldots, N$,

$$
\int_{\mathbb{R}}\left|\hat{w}\left(t_{n}\right)-\hat{c}_{n}\right| \psi_{0}^{\delta} \leq \int_{\mathbb{R}}\left|\hat{w}\left(t_{n-1}\right)-\hat{c}_{n}\right| \psi_{0}^{\delta}+\int_{t_{n-1}}^{t_{n}} \int_{\mathbb{R}} \bar{\Phi}\left(h^{\prime}(t) ; \hat{C}_{N}, \hat{w}\right) \partial_{x} \psi_{0}^{\delta}+M \int_{t_{n-1}}^{t_{n}} \operatorname{dist}_{1}\left(\left(c_{n}^{-}, c_{n}^{+}\right), \mathcal{G}_{\lambda}\left((\hat{h})^{\prime}(t)\right)\right) .
$$

with $\hat{c}_{n}(t, x):=c_{n}^{-} \mathbb{1}_{\mathbb{R}_{-}}(x-\tilde{h}(t))+c_{n}^{+} \mathbb{1}_{\mathbb{R}_{+}}(x-\tilde{h}(t))$ defined for $t \in\left[t_{n-1}, t_{n}\right]$. Summing up the above inequalities in $n$, we find

$-\int_{0}^{T} \int_{\mathbb{R}} \mathcal{T}_{1} \partial_{x} \psi_{0}^{r} \leq \int_{\mathbb{R}}\left|\hat{w}\left(t_{0}\right)-\hat{c}_{1}\right| \psi_{0}^{\delta}-\int_{\mathbb{R}}\left|\hat{w}(T)-\hat{c}_{N}\right| \psi_{0}^{\delta}+\sum_{n=2}^{N} \int_{\mathbb{R}}\left|c_{n}-c_{n-1}\right| \psi_{0}^{\delta}+2 M T \delta+2 M \sum \int_{t_{n-1}}^{t_{n}}\left|h^{\prime}\left(t_{n}\right)-h^{\prime}(t)\right|$,

because $\left(c_{n}^{-}, c_{n}^{+}\right) \in \mathcal{G}_{\lambda}\left(h^{\prime}\left(t_{n}\right)\right)=\mathcal{G}_{\lambda}\left(\hat{h}^{\prime}(t)\right)+\left(h^{\prime}\left(t_{n}\right)-h^{\prime}(t), h^{\prime}\left(t_{n}\right)-h^{\prime}(t)\right)-\left((\tilde{h})^{\prime}(t),(\tilde{h})^{\prime}(t)\right)$. The right-hand side of the above expression is therefore estimated by

$$
C\left(1+\operatorname{Tot} \operatorname{Var} C_{N}^{-}+\operatorname{Tot} \operatorname{Var} C_{N}^{+}\right) \sqrt{\delta}+C \delta+o_{N},
$$

where $o_{N}$ denotes a function of $N$ that converges to 0 as $N$ tends to $+\infty$. This inequality comes from the fact that the rectangle method applied to a continuous function is convergent. We see that this last expression can be bounded by $\varepsilon$, by taking $N$ large enough and then $\delta$ small enough.

In conclusion: given $\varepsilon>0$, 
- we first pick $\eta$ and $\alpha$ so that $C(\eta+\alpha)$ in (48) is less than $\varepsilon / 4$;

- we choose $r$ small enough, $\delta<\sqrt{r}$ and $N$ large enough in order that (48) hold true;

- actually we pick $\delta$ possibly smaller and $N$ possibly greater, to ensure that quantity (49) is less than $\varepsilon / 4$;

- at last, $\delta$ small enough is chosen to ensure that $C \sqrt{\delta}$ in both (45) and (47) is smaller than $\varepsilon / 4$.

Therefore,for any given $\varepsilon>0$ there exists $\delta>0$ such that $\|w-\hat{w}\|_{L^{\infty}\left(0, T ; L^{1}(\mathbb{R})\right)}<\varepsilon$ as soon as $\|h-\hat{h}\|_{E}<\delta$. It remains to notice that

$$
\|u(t, \cdot)-\hat{u}(t, \cdot)\|_{L^{1}(\mathbb{R})}=\|w(t, \cdot)-\hat{w}(t, \cdot)\|_{L^{1}(\mathbb{R})} .
$$

Since $|\tilde{h}(t)| \leq \delta$ and $u \in L^{1}((0, T) \times \mathbb{R})$ admits a finite modulus of continuity for translations, we conclude that $\hat{u}$ tends to $u$ in $L^{1}\left(0, T ; L^{1}(\mathbb{R})\right)$ as $\hat{h} \rightarrow h$ in $E$. This ends the first step of the proof.

$\underline{\text { Step } 2}$

We use the reformulation (39) for $(h, u)$ and $(\hat{h}, \hat{u})$. Due to the $L^{\infty}$ estimate (13), we can choose $\psi$ such that $\psi \equiv 1$ on $\hat{h}([0, T])$ for all relevant $\hat{h}$ belonging to a neighborhood of $h$. Using that $\hat{u}$ tends to $u$ in $L^{1}\left(0, T ; L^{1}(\mathbb{R})\right)$ (previous step) and having in mind the $L^{\infty}$ estimate (13), from (39) we see that $\left(\hat{h}^{*}\right)^{\prime \prime} \rightarrow\left(h^{*}\right)^{\prime \prime}$ weakly in $L^{1}(0, T)$ as $\hat{h} \rightarrow h$ in $E$; therefore $\hat{h}^{*}$ converges strongly to $h^{*}$ in $E$.

Now we are in a position to prove the existence claim, i.e. Theorem 7 . We consider the nonempty closed convex of $C^{1}([0, T])$

$$
\tilde{E}:=\left\{h \in E:\left|h^{\prime}(t)\right| \leq\left[\left|V_{0}\right|+\frac{T}{m}\left(\left\|u_{0}\right\|_{\infty}+\lambda\right)^{2}\right] \exp \left[\frac{2 t}{m}\left(\left\|u_{0}\right\|_{\infty}+\lambda\right)\right] \forall t \in[0, T]\right\} .
$$

Theorem 19 yields that $\mathcal{A}: \tilde{E} \rightarrow E$ is continuous. Moreover, a standard calculation and the a priori $L^{\infty}$ bound (13) show that if $h \in \tilde{E}$, then $h^{*}=\mathcal{A}(h) \in \tilde{E}$ and moreover,

$$
\left\|h^{*}\right\|_{W^{2, \infty}(0, T)} \leq C=C\left(m, \lambda, T,\left|V_{0}\right|,\left\|u_{0}\right\|_{\infty}\right) .
$$

Thus we can apply the Schauder fixed-point theorem in $\tilde{E}$ and eventually get the claim of Theorem 7.

\section{Appendix: $B V$ estimate with wave-front tracking algorithm}

The goal of this appendix is to prove Proposition 13. Unfortunately, the method of proof used in [3] makes it difficult to control carefully the increase of variation of the approximate solutions at the moment of interaction of incoming waves with the interface located at the particle position. Yet we have uniqueness of a solution, therefore we can establish the $B V$ estimate with the help of any other convergent approximation method. Here, in order to prove the Proposition 13, we use the method of wave front tracking.

More precisely, let us take $M_{\lambda} \in \mathbb{N}^{*}$ and let us set $\delta:=\lambda / M_{\lambda}$. We choose $M$ sufficiently large to ensure

$$
0 \leq\left\|u_{0}\right\|_{\infty}+\lambda \leq M \delta
$$

We approximate $u_{0}$ in $L_{\text {loc }}^{1}(\mathbb{R})$ by a piecewise constant function $u_{0}^{\delta}$ taking value in $\delta \mathbb{Z}$, with $\left\|u_{0}^{\delta}\right\|_{\infty} \leq\left\|u_{0}\right\|_{\infty}$ and with TotVar $u_{0}^{\delta}$ converging to TotVar $u_{0}$ as $\delta \rightarrow 0$. Finally, we approximate the map $u \mapsto u^{2} / 2$ by a continuous piecewise affine function $f^{\delta}$, which breakpoints are $k \delta, k \in\{-M, \ldots, M\}$. We take $f^{\delta}(k \delta)=(k \delta)^{2} / 2$ (this yields an even and convex function $f^{\delta}$ strictly increasing on $\mathbb{R}_{+}^{*}$ ). We also consider the discrete germ

$$
\mathcal{G}_{\lambda, \delta}=\mathcal{G}_{\lambda} \cap(\delta \mathbb{Z})^{2} .
$$

Due to the assumption that $\lambda / \delta \in \mathbb{N}^{*}$, every point of $\mathcal{G}_{\lambda}$ is at a distance dist ${ }_{1}$ of at most $\delta$ from the set $\mathcal{G}_{\lambda, \delta}$ (see Fig. 5; pay particular attention to the straight line $\mathcal{G}_{\lambda}^{1}$ that is a part of $\mathcal{G}_{\lambda}$.)

Then we consider the following approximation of (16), (17) (the problem should be interpreted in the way analogous to Definition 2(i)):

$$
\begin{gathered}
\partial_{t} u+\partial_{x}\left(f^{\delta}(u)\right)=0 \text { in } \mathbb{R}^{*} \\
\left(u\left(t, 0^{-}\right), u\left(t, 0^{+}\right)\right) \in \mathcal{G}_{\lambda, \delta} \text { for almost every } t \in \mathbb{R}_{+}, \\
u(0)=u_{0}^{\delta} .
\end{gathered}
$$




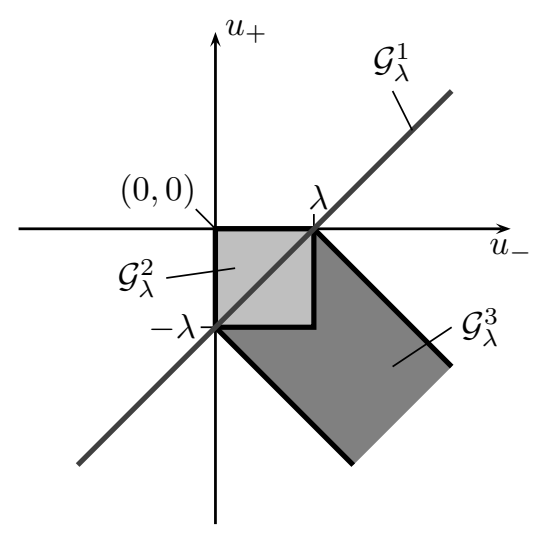

Figure 1: Representation of the admissibility germ $\mathcal{G}_{\lambda}=\mathcal{G}_{\lambda}^{1} \cup \mathcal{G}_{\lambda}^{2} \cup \mathcal{G}_{\lambda}^{3}$ (with the notation of [3]).

The solution $u^{\delta}$ is obtained by juxtaposition of piecewise constant solutions to Riemann problems originated from every point of discontinuity of $u^{\delta}(0, x)=u_{0}^{\delta}(x)$ and from every point of interaction of shocks already present in the solution. These problems are solved in a classical way if the interaction point lies away from the interface $\{x=0\}$; and on the interface, one uses the Riemann solver entirely analogous to the solver described in [13]. Because it is instrumental for the below proof of Proposition 13, we recall the form of this Riemann solver at the end of this section. The only difference between the Riemann solver for the nonlinearity $f^{\delta}$ from the one of [13], $f(u)=u^{2} / 2$, is that the rarefaction waves present in solutions of the cases $(I),(I I),(I I I)$, $(V I)$ and $(V I I)$ are partitioned into a sequence of shocks of amplitude equal to $\delta$. For instance, in case $(I)$ of Theorem 23, if $u_{L}=-a \delta, u_{R}=b \delta$, with $a, b \in \mathbb{N}^{*}, u_{-}=u_{+}=0$ and we insert at the left of the particle the states $u_{L *}^{(a-1)}=-(a-1) \delta, \ldots, u_{L *}^{(1)}=-\delta$ and the right of the particle the states $u_{R *}^{(1)}=\delta, \ldots, u_{R *}^{(b-1)}=(b-1) \delta$. With the above construction, the solution $u=u^{\delta}$ remains a step function with values in $\{-M \delta, \ldots, M \delta\}$ (in particular, notice that the technique of proof of Lemma 9 allows to prove the same $L^{\infty}$ bounds for (50), (51), (52) as for problem (16), (17)).

We need to check that this wave front tracking algorithm gives a global in time solution. To ensure this, we can show that there are only a finite number of interactions between discontinuities. In passing, we will show a uniform in $\delta$ bound on the total variation of these solutions. We consider the two functionals

$$
\Sigma(t):=2 N(t)+N_{a}(t), \quad \text { and } \quad \operatorname{Tot} \operatorname{Var} u^{\delta}(t, \cdot),
$$

where $N(t)$ is the number of fronts at time $t$ and where $N_{a}(t)$ is the number of fronts approaching the particle. We call a front a discontinuity of $u$ at some point $x \neq 0$ and we say it approaches the particle if this discontinuity is at the left of 0 with a positive velocity or at the right of 0 with a negative velocity. Remark that since the initial condition $u_{0}$ has a bounded total variation and since $u_{0}^{\delta}$ takes values in $\delta \mathbb{Z}, N(0)$ is finite. By convention, we assume that $N(\cdot)$ and $N_{a}(\cdot)$ are defined except at the front collision times.

Let us start the analysis with some important remarks: with the above construction of $f^{\delta}$, we notice that a front separating the states $u^{(1)}, u^{(2)}$ is moving with the velocity $\left(u^{(1)}+u^{(2)}\right) / 2$. We deduce from this remark that any collision in the fluid of several fronts results in at most one front. In particular, in a collision between fronts, $N$ is decreasing by at least one, and $N_{a}$ is increasing at most by one. Consequently, $\Sigma$ is decreasing for any collision in the fluid. It is also simple to show that TotVar $u(t, \cdot)$ is non-increasing during the collision. It remains to analyze the evolution of $\Sigma$ and $\operatorname{Tot} \operatorname{Var} u(t, \cdot)$ for a collision between one or several fronts and the particle (at $x=0)$.

More precisely, let us consider an interaction that will occur at time $t$ between the particle and fronts at the left of the particle separating, before $t$, the states

$$
u_{L}=: u_{*}^{(1)}, \ldots, u_{*}^{(m)}:=u_{-},
$$

and fronts at the right of the particle separating, before $t$, the states

$$
u_{+}=: u_{*}^{(m+1)}, u_{*}^{(m+2)}, \ldots, u_{*}^{(m+n)}:=u_{R} .
$$


Here above, it is assumed that the position of the discontinuity separating $u_{*}^{(k)}$ and $u_{*}^{(k+1)}$ is an increasing function of $k$. Note that since we consider the case of collision between at least one front and the particle, $\max \{n, m\} \geq 2$. After the collision, we are reduced to solve a Riemann problem with the states $\left(u_{L}, u_{R}\right)$.

Remark 20. After the initial time, for any front separating the states $u^{(1)}$ on its left and $u^{(2)}$ on its right, we have either $u^{(1)}>u^{(2)}$ or $u^{(1)}=u^{(2)}-\delta$. As a consequence, if $u_{L}<0$, then there are no fronts satisfying (53) (with $m \geq 2$ ) and colliding the particle: we would have $u_{L} \leq-\delta$ and $u_{*}^{(2)} \leq 0$ which is impossible, because in this case the wave speed of the front separating $u_{L}$ and $u_{*}^{(2)}$ would be negative. Symmetrically, if $u_{R}>0$, then there are no fronts satisfying (54) with $n \geq 2$ and colliding the particle.

Remark 21. Note that by the very definition of the solutions, $\left(u_{-}, u_{+}\right) \in \mathcal{G}_{\lambda, \delta}$.

At the instant of such a collision, one has to solve the Riemann problem with the states $u_{L}$ and $u_{R}$. The solution being given by Theorem 23 , let us analyze each of the eight cases (according to the values of $u_{L}$, $u_{R}$ and $\lambda$ ) of this theorem. It is sufficient to consider only the evolution of $\Sigma$ and of TotVar $u$ in a neighborhood of the particle, which means that we can assume that the only fronts in the fluid are the fronts in (53) and (54) and the fronts produced after the collision of these fronts with the particle. For simplicity, we write $N^{-}, N_{a}^{-}$, $\Sigma^{-}$and $T V^{-}$(respectively $N^{+}, N_{a}^{+}, \Sigma^{+}$and $T V^{+}$) for the value of $N, N_{a}, \Sigma$ and TotVar $u$ at $t^{-}$(respectively at $t^{+}$). Our aim is to show that for every case of Theorem 23 we have

$$
\Sigma^{+}<\Sigma^{-} \text {and } T V^{+} \leq T V^{-}
$$

The above relation yields that there is only a finite number of collisions between fronts and the particle and that the total variation of each solution $u^{\delta}$ is bounded uniformly with respect to $\delta$.

1. Case $(I): u_{L} \leq 0$ and $u_{R} \geq 0$. In virtue of Remark 20, if $u_{L}<0$ then $u_{L}=u_{-}<0$. In that case, $\left(u_{-}, u_{+}\right) \in \mathcal{G}_{\lambda, \delta}$ implies $u_{+}=u_{-}-\lambda<0$. This last equality and Remark 20 yield that $u_{R}=0$. We deduce that

$$
N^{-} \geq \frac{-u_{L}+\lambda}{\delta}, \quad N_{a}^{-} \geq 1, \quad T V^{-} \geq 2 \lambda-u_{L}
$$

From Theorem 23, we have

$$
N^{+}=\frac{-u_{L}}{\delta}, \quad N_{a}^{+}=0, \quad T V^{+}=-u_{L}
$$

The above relations imply $(55)$. The case $u_{L}=0, u_{R}>0$ can be done in a similar way and we skip it. In the case $u_{L}=u_{R}=0$, Theorem 23 yields

$$
N^{+}=0, \quad N_{a}^{+}=0, \quad T V^{+}=0,
$$

and thus (55) holds.

2. Case $(I I): u_{L}<0$ and $u_{R}<0$ and $u_{R}>-\lambda$. From Remark 20, we deduce $u_{L}=u_{-}<0$. In that case, $\left(u_{-}, u_{+}\right) \in \mathcal{G}_{\lambda, \delta}$ implies $u_{+}=u_{L}-\lambda=-\lambda<u_{R}$. We deduce that

$$
N^{-} \geq \frac{u_{R}-u_{L}+\lambda}{\delta}, \quad N_{a}^{-} \geq 1, \quad T V^{-} \geq 2 \lambda+u_{R}-u_{L} .
$$

From Theorem 23, one has

$$
N^{+}=\frac{-u_{L}}{\delta}=0, \quad N_{a}^{+}=0, \quad T V^{+}=-u_{L}-u_{R}=T V^{-} .
$$

The above relations imply (55).

3. Case $(I I I): u_{L} \leq u_{R}+\lambda<0$. This yields $u_{L} \leq 0$. If $u_{L}=0$, then $u_{R}=-\lambda$. In that case,

$$
N^{-} \geq 1, \quad N_{a}^{-} \geq 1, \quad T V^{-} \geq u_{L}-u_{R}=-u_{R},
$$

while Theorem 23 gives

$$
N^{+}=0, \quad N_{a}^{+}=0, \quad T V^{+}=-u_{R} .
$$

This yields (55) in this case. 
If $u_{L}<0$, then $u_{L}=u_{-}$and $u_{+}=u_{L}-\lambda$. In that case,

$$
N^{-} \geq \frac{u_{R}-u_{L}+\lambda}{\delta}, \quad N_{a}^{-} \geq 1, \quad T V^{-} \geq 2 \lambda+u_{R}-u_{L}
$$

while Theorem 23 gives

$$
N^{+}=\frac{u_{R}-u_{L}+\lambda}{\delta}, \quad N_{a}^{+}=0, \quad T V^{+}=2 \lambda+u_{R}-u_{L}
$$

This yields (55) in this case. Note that it is possible to have $N^{-}=N^{+}$and $T V^{-}=T V^{+}$. In this case, the presence of $N_{a}$ in $\Sigma$ is essential in our analysis.

4. Case $(I V): u_{R}<u_{L}-\lambda$ and $u_{R}<-u_{L}-\lambda$. In that case,

$$
N^{-} \geq 1, \quad N_{a}^{-} \geq 1, \quad T V^{-} \geq u_{L}-u_{R},
$$

while Theorem 23 gives

$$
N^{+}=1, \quad N_{a}^{+}=0, \quad T V^{+}=u_{L}-u_{R} .
$$

This yields (55) in this case. As in the previous case, it is possible to have $N^{-}=N^{+}$and $T V^{-}=T V^{+}$ and we need $N_{a}$ to obtain the inequality of $\Sigma$ in (55).

5. Case $(V): u_{L} \geq 0$ and $u_{R} \leq 0$ and $u_{R} \geq-u_{L}-\lambda$ and $u_{R} \leq-u_{L}+\lambda$. In that case,

$$
N^{-} \geq 1, \quad N_{a}^{-} \geq 1, \quad T V^{-} \geq u_{L}-u_{R},
$$

and Theorem 23 gives

$$
N^{+}=0, \quad N_{a}^{+}=0, \quad T V^{+}=u_{L}-u_{R}
$$

The remaining cases are obtained in a symmetrical way.

Proof. (of Prop. 13) In the above construction, we obtained a globally defined sequence $\left(u^{\delta}\right)_{\delta \in \mathbb{R}_{+}^{*}}$ of solutions to $(50)-(52)$ such that $\operatorname{Tot} \operatorname{Var} u^{\delta}(t, \cdot)$ is non-increasing for $t \in(0, T]$. Moreover, during the initial interaction in the wave-front tracking algorithm, the variation may increase by at most $2 \operatorname{dist}_{1}\left(\left(u_{0}^{\delta}\left(0^{-}\right), u_{0}^{\delta}\left(0^{+}\right)\right), \mathcal{G}_{\lambda, \delta}\right)$. Therefore

$$
\lim _{\delta \rightarrow 0}\left(\sup _{t \in[0, T]} \operatorname{Tot} \operatorname{Var} u^{\delta}\right) \leq \operatorname{Tot} \operatorname{Var} u_{0}+2 \operatorname{dist}_{1}\left(\left(u_{0}\left(0^{-}\right), u_{0}\left(0^{+}\right)\right), \mathcal{G}_{\lambda}\right) .
$$

Notice that the space-time compactness in the sense of pointwise a.e. convergence is ensured by the uniform $B V$ bound in space: along with the uniform $L^{\infty}$ bound and equation (50), it ensures a $B V$ bound in time. By the lower semi-continuity of the total variation, there exists a limit $u$ of (a subsequence of) $\left(u^{\delta}\right)_{\delta}$ that verifies the variation bound (18) of Proposition 13. Further, the functions $u^{\delta}$ can be seen as approximate solutions of (16),(17). Writing the entropy formulation of the kind (9), we can pass to the limit in the entropy formulation and inherit property (9) for the accumulation point $u$ of our sequence, as $\delta \rightarrow 0$.

In conclusion, we reproduce the result of [13] used here above. We need only the particular result corresponding to the case when the velocity of the particle is 0 . Notice that the solutions of the Riemann problem are entropy solutions in the sense of Definition 2 (or Definition 5).

Theorem 22. Consider the Riemann problem for (16). For every pair $\left(u_{L}, u_{R}\right) \in \mathbb{R}^{2}$, the solution with the Riemann datum $u_{0}(x)=u_{L} \mathbb{1}_{\mathbb{R}^{-}}(x)+u_{R} \mathbb{1}_{\mathbb{R}_{+}}(x)$ is given by the formula $u(t, x)=\mathbb{U}(x / t)$ with $\mathbb{U}$ described by the expressions below, where Id is the identity map.

1. If $u_{L} \leq 0$ and $u_{R} \geq 0$,

$$
(I) \mathbb{U}(\xi)= \begin{cases}u_{L} & \text { if } \xi \leq u_{L} \\ \operatorname{Id}(\xi) & \text { if } u_{L}<\xi \leq u_{R} \\ u_{R} & \text { if } u_{R}<\xi\end{cases}
$$

$$
\text { 2.If } u_{L}<0 \text { and } u_{R}<0 \text { and } u_{R}>-\lambda \text {, }
$$$$
(I I) \quad \mathbb{U}(\xi)= \begin{cases}u_{L} & \text { if } \xi \leq u_{L} \\ \operatorname{Id}(\xi) & \text { if } u_{L}<\xi \leq 0 \\ u_{R} & \text { if } 0<\xi\end{cases}
$$ 
3. If $u_{R}<-\lambda$ and $u_{R} \geq u_{L}-\lambda$,

$$
(I I I) \mathbb{U}(\xi)= \begin{cases}u_{L} & \text { if } \xi \leq u_{L}, \\ \operatorname{Id}(\xi) & \text { if } u_{L}<\xi \leq u_{R}+\lambda, \\ u_{R}+\lambda & \text { if } u_{R}+\lambda<\xi \leq 0, \\ u_{R} & \text { if } 0<\xi .\end{cases}
$$

5. If $u_{L} \geq 0$ and $u_{R} \leq 0$

and $u_{R} \geq-u_{L}-\lambda$ and $u_{R} \leq-u_{L}+\lambda$,

$$
(V) \mathbb{U}(\xi)= \begin{cases}u_{L} & \text { if } \xi \leq 0, \\ u_{R} & \text { if } 0<\xi .\end{cases}
$$

7. If $u_{L}>\lambda$ and $u_{R} \geq u_{L}-\lambda$,

$$
(V I I) \mathbb{U}(\xi)= \begin{cases}u_{L} & \text { if } \xi \leq 0, \\ u_{L}-\lambda & \text { if } 0<\xi \leq u_{L}-\lambda, \\ \operatorname{Id}(\xi) & \text { if } u_{L}-\lambda<\xi \leq u_{R}, \\ u_{R} & \text { if } u_{R}<\xi\end{cases}
$$

4. If $u_{R}<u_{L}-\lambda$ and $u_{R}<-u_{L}-\lambda$,

$$
\mathbb{U}(\xi)= \begin{cases}u_{L} & \text { if } \xi \leq \frac{u_{L}+u_{R}+\lambda}{2}, \\ u_{R}+\lambda & \text { if } \frac{u_{L}+u_{R}+\lambda}{2}<\xi \leq 0, \\ u_{R} & \text { if } 0<\xi\end{cases}
$$

6. If $u_{L}>0$ and $u_{L} \leq \lambda$ and $u_{R}>0$,

$$
\mathbb{U}(\xi)= \begin{cases}u_{L} & \text { if } \xi \leq 0 \\ \operatorname{Id}(\xi) & \text { if } 0<\xi \leq u_{R} \\ u_{R} & \text { if } u_{R}<\xi\end{cases}
$$

8. If $u_{R}<u_{L}-\lambda$ and $u_{R}>-u_{L}+\lambda$,

$$
\mathbb{U}(\xi)= \begin{cases}u_{L} & \text { if } \xi \leq 0, \\ u_{L}-\lambda & \text { if } 0<\xi \leq \frac{u_{L}+u_{R}-\lambda}{2}, \\ u_{R} & \text { if } \frac{u_{L}+u_{R}-\lambda}{2}<\xi\end{cases}
$$

It is not difficult to see that for the discretized problem

$$
\partial_{t} u+\partial_{x} f^{\delta}(u)=-\lambda u \delta_{0}(x),
$$

we have a similar result that is stated below. Let us note that a crucial ingredient for this theorem is that $\lambda / \delta \in \mathbb{N}^{*}$.

Theorem 23. Consider the Riemann problem for (56) $\partial_{t} u+\partial_{x} f^{\delta}(u)=-\lambda u \delta_{0}(x)$. For every pair $\left(u_{L}, u_{R}\right) \in$ $(\delta \mathbb{Z})^{2}$, the solution with the Riemann datum $u_{0}(x)=u_{L} \mathbb{1}_{\mathbb{R}^{-}}(x)+u_{R} \mathbb{1}_{\mathbb{R}_{+}}(x)$ is given by the formula $u(t, x)=$ $\mathbb{U}(x / t)$ with $\mathbb{U}$ described by the same expressions as in Theorem 22 with Id replaced by the function $I^{\delta}$ which is the discretized identity function:

$$
I^{\delta}(\xi)=\sum_{k \in \mathbb{Z}} k \delta \mathbb{1}_{[(k-1 / 2) \delta,(k+1 / 2) \delta)}(\xi) .
$$

Acknowledgments The work of B.A. was partially supported by ANR Grant CoToCoLa. The work of F.L., N.S. and T.T. was partially supported by ANR Grant CISIFS (09-BLAN-0213-02). The work of F.L. was partially supported by ANR Grants INTOCS (08-JCJC-0132-01)

\section{References}

[1] Boris Andreianov, Kenneth Hvistendahl Karlsen, and Nils Henrik Risebro. A theory of $L^{1}$-dissipative solvers for scalar conservation laws with discontinuous flux. Arch. Ration. Mech. Anal., 201(1):26-86, 2011.

[2] Boris Andreianov, Frédéric Lagoutière, Nicolas Seguin, and Takéo Takahashi. Small solids in an inviscid fluid. Netw. Heterog. Media, 5(3):385-404, 2010.

[3] Boris Andreianov and Nicolas Seguin. Well-posedness of a singular balance law. Discr. Cont. Dyn. Syst. A, 32(6):1939-1964, 2012.

[4] Raul Borsche, Rinaldo M. Colombo, and Mauro Garavello. Mixed systems: ODEs - balance laws. J. Differential Equations, 252(3):2311-2338, 2012.

[5] François Bouchut and Benoît Perthame. Kružkov's estimates for scalar conservation laws revisited. Trans. Amer. Math. Soc., 350(7):2847-2870, 1998.

[6] Clément Cancès and Thierry Gallouët. On the time continuity of entropy solutions. J. Evol. Equ., 11(1):43$55,2011$. 
[7] Constantine M. Dafermos. Polygonal approximations of solutions of the initial value problem for a conservation law. J. Math. Anal. Appl., 38:33-41, 1972.

[8] Helge Holden and Nils Henrik Risebro. Front tracking for hyperbolic conservation laws, volume 152 of Applied Mathematical Sciences. Springer-Verlag, New York, 2002.

[9] Eli L. Isaacson and J. Blake Temple. Convergence of the $2 \times 2$ Godunov method for a general resonant nonlinear balance law. SIAM J. Appl. Math., 55(3):625-640, 1995.

[10] Kenneth Hvistendahl Karlsen and Nils Henrik Risebro. On the uniqueness and stability of entropy solutions of nonlinear degenerate parabolic equations with rough coefficients. Discrete Contin. Dyn. Syst., 9(5):10811104, 2003.

[11] Stanislav N. Kružkov. First order quasilinear equations with several independent variables. Mat. Sb. (N.S.), $81(123): 228-255,1970$.

[12] Young-Sam Kwon and Alexis Vasseur. Strong traces for solutions to scalar conservation laws with general flux. Arch. Ration. Mech. Anal., 185(3):495-513, 2007.

[13] Frédéric Lagoutière, Nicolas Seguin, and Takéo Takahashi. A simple 1D model of inviscid fluid-solid interaction. J. Differential Equations, 245(11):3503-3544, 2008.

[14] Alain Yves Le Roux. Riemann solvers for some hyperbolic problems with a source term. In Actes du 30ème Congrès d'Analyse Numérique: CANum '98 (Arles, 1998), volume 6 of ESAIM Proc., pages 75-90 (electronic). Soc. Math. Appl. Indust., Paris, 1999.

[15] Magali Lécureux-Mercier. Improved stability estimates for general scalar conservation laws. Journal of Hyperbolic Differential Equations, 8(4):727-757, 2011.

[16] Pierre-Louis Lions, Benoit Perthame, and Eitan Tadmor. Kinetic formulation of the isentropic gas dynamics and p-systems. Comm. Math. Phys., 163(2):415-431, 1994.

[17] Evgueni Yu. Panov. Existence of strong traces for quasi-solutions of multidimensional conservation laws. J. Hyperbolic Differ. Equ., 4(4):729-770, 2007.

[18] Evgueni Yu. Panov. Existence and strong pre-compactness properties for entropy solutions of a first-order quasilinear equation with discontinuous flux. Arch. Ration. Mech. Anal., 195(2):643-673, 2010.

[19] Nicolas Seguin and Julien Vovelle. Analysis and approximation of a scalar conservation law with a flux function with discontinuous coefficients. Math. Models Methods Appl. Sci., 13(2):221-257, 2003.

[20] Alexis Vasseur. Well-posedness of scalar conservation laws with singular sources. Methods Appl. Anal., $9(2): 291-312,2002$. 